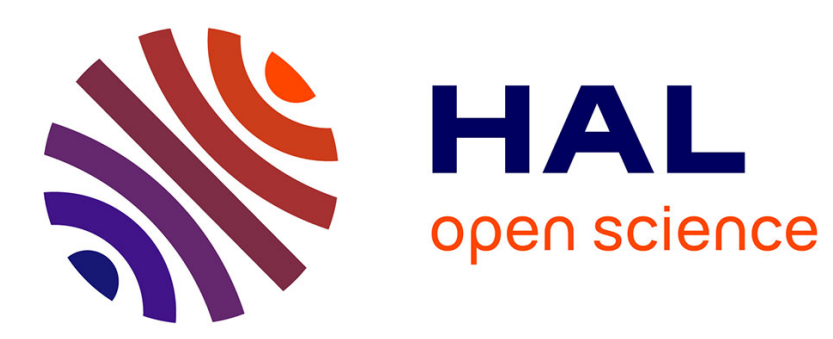

\title{
Thermodynamics of small systems with conformational transitions: The case of two-state freely jointed chains with extensible units
}

Manon Benedito, Stefano Giordano

\section{- To cite this version:}

Manon Benedito, Stefano Giordano. Thermodynamics of small systems with conformational transitions: The case of two-state freely jointed chains with extensible units. Journal of Chemical Physics, 2018, 149 (5), pp.054901. 10.1063/1.5026386 . hal-02337978

\section{HAL Id: hal-02337978 \\ https://hal.science/hal-02337978}

Submitted on 5 Oct 2020

HAL is a multi-disciplinary open access archive for the deposit and dissemination of scientific research documents, whether they are published or not. The documents may come from teaching and research institutions in France or abroad, or from public or private research centers.
L'archive ouverte pluridisciplinaire HAL, est destinée au dépôt et à la diffusion de documents scientifiques de niveau recherche, publiés ou non, émanant des établissements d'enseignement et de recherche français ou étrangers, des laboratoires publics ou privés. 


\section{Thermodynamics of small systems with conformational transitions: the case of two-state freely jointed chains with extensible units}

Manon Benedito ${ }^{1}$ and Stefano Giordano ${ }^{1, \text { a) }}$

Institute of Electronics, Microelectronics and Nanotechnology - UMR 8520, Univ. Lille, CNRS, Centrale Lille, ISEN, Univ. Valenciennes, LIA LICS/LEMAC, F-59000 Lille,

France

(Dated: 12 June 2018)

Several experimental methods are usually applied for stretching single molecules and provide valuable insights about the static and dynamic responses induced by externally applied forces. This analysis is even more important for macromolecules exhibiting conformational transitions, thereby corresponding to folding/unfolding processes. With the aim of introducing the statistical mechanics of such phenomena, we apply here the spin variables approach, based on a set of discrete quantities able to identify the folded or unfolded state of the chain units. Firstly, we obtain the macroscopic thermodynamics of the chain from its microscopic description. For small systems, far from the thermodynamic limit, this result depends on the applied boundary condition (e.g., isometric or isotensional), which corresponds to the considered statistical ensemble. Then, we develop the theory for the two-state extensible freely jointed chain, where the elastic constant of the units, a property often neglected, plays a central role in defining the force-extension curve. For this system, the partition function of the isometric ensemble can be written in closed form in terms of the natural generalization of the Hermite polynomials, obtained by considering negative indices. These results are relevant for the interpretation of stretching experiments, operated from the entropic regime up to the unfolding processes.

\section{INTRODUCTION}

The advent of single-molecule experiments (force spectroscopy) allowed the direct quantification of the elasticity and the dynamical properties of individual macromolecules. ${ }^{1-3}$ For the first time, these experimental activities probed the thermodynamics and the statistical mechanics of small systems. ${ }^{4,5}$ The properties of macromolecules with and without conformational transitions have been investigated through different devices, such as atomic-force microscopes (AFM, in the range 10$10^{4} \mathrm{pN}$ ), laser optical tweezers (LOT, in the range $0.1-$ $200 \mathrm{pN}$ ) and magnetic tweezers (MT, in the range 0.0110pN). ${ }^{6-11}$ Such approaches have been largely employed for proteins, ${ }^{12-16} \mathrm{RNA},{ }^{17,18}$ and DNA. ${ }^{19-24}$ Also, microelectro-mechanical systems (MEMS), and in particular silicon nanotweezers (SNT), ${ }^{25}$ have been applied to evaluate the DNA mechanical degradation under ionizing radiation. ${ }^{26,27}$

Through these techniques, it is possible to explore the whole stretching behavior composed of entropic, enthalpic, unfolding and over-stretching regimes. In particular, the unfolding of the units of a chain, governed by the conformational transition between two (or more) states, is an important process, e.g., observed in polypeptides and nucleic acids. It is based on transformations of the units of the chains, which modify their length and elastic properties. These transitions can be taken into account by means of a potential energy exhibiting two (or more) minima corresponding to the stable states or configurations.

a)Electronic mail: stefano.giordano@iemn.univ-lille1.fr

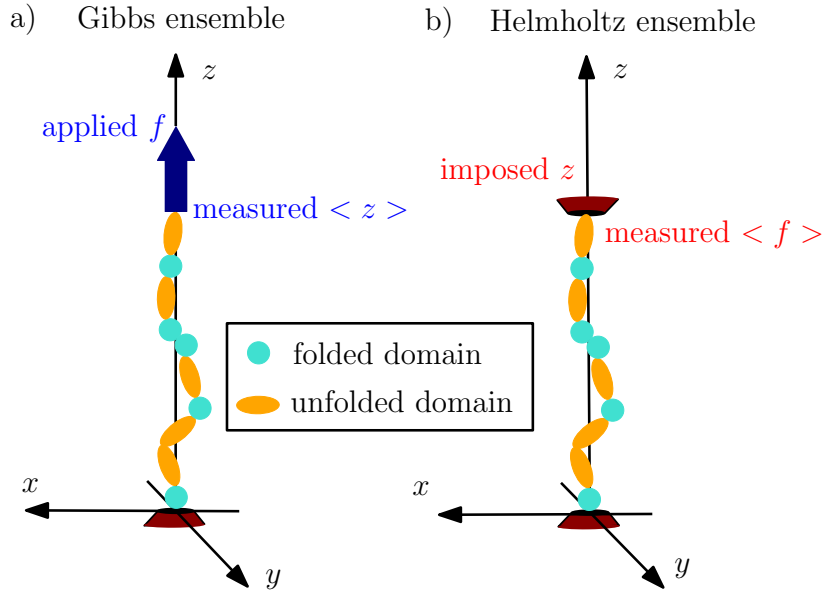

FIG. 1. Single-molecule stretching experiments conducted within the Gibbs (a) and the Helmholtz (b) ensembles. Each domain of the chain may experience a conformational transition between folded (length $\ell$ ) and unfolded (length $\chi \ell$ ) states.

Today, many efforts are devoted to the application of theoretical physics methods to the complexity of the biological context. ${ }^{28}$ Accordingly, models for biopolymer chains introduce a specific Hamiltonian of the system and proceed by calculating the pertinent partition function and, eventually, the thermal and mechanical macroscopic properties. ${ }^{29-34}$. Classical theoretical developments for macromolecules without configurational transitions are given by the freely-jointed chain (FJC) model, ${ }^{33,35}$ the worm-like chain (WLC) model, ${ }^{20,30,36}$ and many other generalized approaches. ${ }^{37-41}$ Conversely, for macromolecules undergoing folding/unfolding transformations, the models must consider the boundary condition imposed to the system (see Fig.1 for details). In- 
deed, the isotensional experiments (at constant applied force), typically performed with soft devices, correspond to the Gibbs statistical ensemble (Fig.1a), and the isometric experiments (at prescribed displacement), performed by hard devices, represent a realization of the Helmholtz statistical ensemble (Fig.1b). ${ }^{42-45}$ From the point of view of the force-extension response, isotensional conditions (Gibbs ensemble) lead to a plateau-like response with a threshold force characterizing the synchronized unfolding of all chain units (see for example Fig.3 in Section III). This point can be interpreted through a cooperative process, which induces the simultaneous transition of the macromolecular domains. ${ }^{21,22,46-48} \mathrm{On}$ the contrary, the force-extension curve under isometric conditions (Helmholtz ensemble) shows a sawtooth-like shape, proving that the units unfold sequentially in reaction to the increasing extension (see for example Fig.5 in Section IV). This behavior is therefore interpreted through a non-cooperative process, with a progressive unfolding of the units. ${ }^{12,14,49-51}$

The differences in the force-extension curves mentioned above (Helmholtz versus Gibbs ensemble) can be measured when the thermodynamic limit is not satisfied (small number of monomers). On the other hand, whenever the number of units is very large (ideally, when it approaches infinity), it is important to remark that the statistical ensembles become equivalent and they are described by the same constitutive force-extension response. ${ }^{37,52-55}$

In order to get a wider comprehension of this complex scenario, several theoretical approaches have been so far proposed in literature. Concerning the Gibbs ensemble, the plateau-like response observed for doublestranded DNA has been largely investigated with thermodynamic approaches, statistical mechanics and molecular dynamics simulations. ${ }^{47,56-60}$ A similar response has been also observed for long polysaccharides (e.g.,dextran) and modeled through a continuous two-state model. ${ }^{42,49,61}$

In a like manner, theoretical developments have been adopted to model the sawtooth-like response observed within the Helmholtz ensemble. Theories for titin, RNA hairpins and other macromolecules have been elaborated through Landau-like free energies, first-order phase transition, Langevin equations, and Ising models. ${ }^{62-67}$ A more general point of view about two-state systems driven by hard or soft devices can be found in the mechanical literature concerning discrete systems with multi-basin energy landscapes, Fermi-Pasta-Ulam chains of bistable elements, and structures undergoing discrete phase transformations. ${ }^{68-71}$

We present in this paper a general methodology to cope with the problem of analysing the response of a system composed of two-state units and subjected to different boundary conditions. While in the first part of the paper, we briefly introduce the statistical mechanics of this system and its macroscopic thermodynamic properties, in the second part, we obtain the complete solution for a specific case, namely the two-state freely jointed chain with extensible units. The main idea consists in associating to each unit a discrete variable (or spin variable), able to define the state of the unit itself. Such a variable represents a sort of "bit", assuming the values 0 and 1 for the folded and the unfolded states, respectively. The set of spin variables assume therefore the character of dynamic variables, thus belonging to the extended phase space of the system. From the historical point of view, the first biophysical model based on a discrete quantity, similar to a spin variable, has been performed to predict the response of skeletal muscles. ${ }^{72,73}$ This method has been recently applied to different allosteric systems and macromelecular chains as well. ${ }^{74-77}$ The introduction of the spin variables is advantageous since strongly facilitates the calculation of the partition functions, preserving at the same time a good accuracy of results. ${ }^{76}$ Briefly, an arbitrary potential function composed of two minima, can be substituted by two quadratic potentials and the switching between them is controlled by the corresponding spin variable. This method works for both Gibbs and Helmholtz ensembles, allowing to draw direct comparisons between isotensional and isometric conditions. We remark that the spin variables approach can be only adopted for systems at thermodynamic equilibrium. As a matter of fact, the quadratic potentials and the associated spin variables are not sufficient to describe the dynamics since the characteristic relaxation times strongly depend on the energy barriers between the potential wells. This is a well-known result, encoded within the Kramers rate formula, originally formulated to study chemical reactions, ${ }^{78}$ and recently generalized for arbitrary systems with nonconvex energy landscapes. ${ }^{79,80}$

Concerning the two-state freely jointed chain with extensibility, we have here generalized a recent result obtained for the same system without extensibility. ${ }^{76}$ It is important to remark that the finite elastic constant of the units plays a crucial role in defining the force-extension response in both isotensional and isometric ensembles. In particular, within the Helmholtz ensemble, the peak-topeak force of the sawtooth-like curve strongly depends on the elastic constant. Since this quantity is often considered as the main output of the force spectroscopy measurements, the finite value of the elastic constant can not be neglected and its effect is here studied in detail. From the mathematical point of view, the most difficult issue concerns the calculation of the Helmholtz partition function. Eventually, its closed form expression has been found in terms of the Hermite polynomials, suitably generalized to negative indices.

\section{THERMODYNAMICS OF CHAINS WITH CONFORMATIONAL TRANSITIONS}

We introduce here a general methodology to approach the problem of describing the conformational transitions in chains undergoing typical folding/unfolding processes. It means that each domain or unit of the chain may be 
in two states corresponding to different chemical conformations. The potential energy of the system is therefore constituted of two wells, which can be explored during the dynamics of the system. To simplify the description of the system, considered at thermodynamic equilibrium, it is often convenient to introduce discrete variables or spins, which are able to indicate what basin is explored for each unit of the chain. Consequently, the more general form of the Hamiltonian for such a system can be written as

$$
H=\sum_{i=1}^{N} \frac{\vec{p}_{i} \cdot \vec{p}_{i}}{2 M}+U\left(\vec{r}_{1}, \ldots, \vec{r}_{N}, S_{1}, \ldots, S_{N}\right)-\sum_{i=1}^{N} \mu_{i} S_{i},(1)
$$

where $S_{i} \in\{0,1\}$ are the spin variables $\forall i=1, \ldots, N$ introduced in the potential energy $U$. We say that the domain is folded (it is in the first energy well) when $S_{i}=0$ and that the domain is unfolded (it is in the second energy well) when $S_{i}=1$. The first term represents the kinetic energy $\left(\vec{p}_{i}\right.$ are the linear momenta of the units and $M$ is their mass) and the second term represents the potential energy depending on the units positions $\vec{r}_{i}$ and on the spins $S_{i}$. Finally, the third term represents the effect of the external chemical environment on the state of the domains: if $\mu_{i}>0$, the unfolded state is favored, and conversely, if $\mu_{i}<0$, the folded state is favored. From the experimental point of view, the chemical potentials $\mu_{i}$ may mimic the presence of some chemical substances, which have the capability to induce or to impede the units unfolding. An interesting example concerns the effect of ethidium molecules on the force-induced melting of DNA. ${ }^{81}$ As discussed below in our model, the chemical potential is able to shift the effective energy jump between folded and unfolded states of a given bistable system. This exactly corresponds to the experimental observations in response to an increasing concentration of ethidium, which reported an increase of the DNA transition force and of the effective energy jump between states before and after the transition. ${ }^{81}$

It is important to remark that the introduced Hamiltonian function corresponds to a coarse-graining approach, applied to the actual molecular architecture of the system. Indeed, the concept of units above introduced corresponds to a specific ensemble of atoms with a well-defined behavior, summarized within the coarse-grained potential energy. Of course, we could have considered an exact Hamiltonian with a very large number of degrees of freedom (all the atomic coordinates and momenta as, e.g., introduced in classical molecular dynamics), but we propose a simplification to provide analytic developments and corresponding physical interpretations. Therefore, when we mention a physical property of one unit (elastic constant or others), we have to keep in mind that it represents the collective response of the atoms of the unit, and not a real or intrinsic property of an exact Hamiltonian. Then, these properties of units may be considered temperature-dependent being defined at the mesoscopic scale. This is similar to the expansion of the Landau free energy used to describe critical phenomena, where the coefficients depend on temperature for the same reason. The coarse-graining approach also leads to the following issue in writing Eq.(1): since the extremities of a given units $\vec{r}_{i-1}$ and $\vec{r}_{i}$ are associated to the momenta $\vec{p}_{i-1}$ and $\vec{p}_{i}$, it means that we assume the mass concentrated at points $\vec{r}_{i}$, while in the real case the mass is distributed between $\vec{r}_{i-1}$ and $\vec{r}_{i}$ on all the atoms composing the unit. This is a typical approximation used to describe a onedimensional chain, ${ }^{82}$ but it should be kept in mind since each unit is finally identified by the two positions of extremities and by one spin variable.

As an example, a concrete case can be described by the following potential energy

$$
\begin{aligned}
U= & -\sum_{i=1}^{N-1} \lambda_{i}\left(2 S_{i}-1\right)\left(2 S_{i+1}-1\right) \\
& +\sum_{i=1}^{N} \frac{1}{2} k\left(S_{i}\right)\left[\left\|\vec{r}_{i}-\vec{r}_{i-1}\right\|-\ell\left(S_{i}\right)\right]^{2}+\sum_{i=1}^{N} v\left(S_{i}\right) \\
& +\sum_{i=1}^{N-1} \frac{1}{2} \kappa\left(S_{i}\right)\left(\vec{t}_{i+1}-\vec{t}_{i}\right)^{2}
\end{aligned}
$$

where: (i) the first line represents an Ising interaction among the states of the units $\left(\lambda_{i}\right.$ being the interaction coefficients and $2 S_{i}-1= \pm 1$ if $S_{i}=0,1$ ), (ii) the second line represents the spring-like behavior of the twostate domains $(k, \ell$ and $v$ are the elastic constant, the equilibrium length and the basal energy, respectively, of the states), and (iii) the third line represents a wormlike-chain interaction among the units $(\kappa$ is the bending constant related to the persistence length $L_{p}=\frac{\ell \kappa}{k_{B} T}$ and $\left.\overrightarrow{t_{i}}=\left(\vec{r}_{i}-\vec{r}_{i-1}\right) /\left\|\vec{r}_{i}-\vec{r}_{i-1}\right\|\right)$.

In the following sections, we will analyze in detail the case without Ising and worm-like-chain interactions. Anyway, it is important to add some comments concerning these kind of interactions in order to understand the relation with recent experimental activities. The Ising scheme represents an interaction among the spin variables, with two adjacent units favored to be in the same state if $\lambda_{i}>0$ or favored to be in opposite states if $\lambda_{i}<0$. The typical case arising in protein structures corresponds to a positive interaction and allows the interpretation of cooperativity of the folding-unfolding process. ${ }^{83}$ An interesting example of cooperativity in forced unfolding concerns the case of tandem repeats in red cell spectrin. ${ }^{84}$ AFM-measured force-extension curves of thousands of constructs with few repeats of spectrin have shown a significant statistic of tandem repeat unfolding processes. It means that two adjacent units unfold at the same time (cooperatively), thus generating a single force peak in the observed response. ${ }^{84}$ The mechanism at the base of this transition lies in the strongly interconnected repeats, which unfold in synchrony. This is the typical phenomenon modeled by an Ising model, as introduced in Eq.(2). Also, a similar observation has been made on Filamin A, where domain-domain interactions lead to a hierarchy of unfolding forces that may be studied by an 
Ising scheme. ${ }^{85}$ An interesting system for studying interactions and cooperativity is given by the ankyrin repeat proteins. They present, contrarily to globular proteins, very few long-range contacts and therefore only local interactions are present. ${ }^{86}$ However, these structures show a high degree of folding cooperativity, and are therefore very stimulating for analyzing the folding-unfolding process. In this system, it is relatively easy to add or remove units, thus generating ideal models to compare experiments and theories. ${ }^{86}$ We also note that the interactions among domains may be affected by chemical substances, which finally modify the folding pathway. As an example, we can cite the effects of chaperones on maltose binding proteins (MBP), recently observed by optical tweezers measurements. ${ }^{87,88}$ From the theoretical point of view, it means that the Ising interaction coefficients may depend on the external chemical conditions.

Concerning the WLC scheme, we recall that it represents a geometric interaction, which provides an elastic flexibility to a chain of objects, trying to align them along the same direction. As an example, in Eq.(2), this form of interaction has been introduced among the units of the chain. It means that the situation with aligned units is energetically favored. Nevertheless, it is interesting to note that in most of protein structures, the WLC scheme can be used to model the force-extension behavior of each unit. It means that it should be implemented at the level of the exact Hamiltonian (at atomic and/or molecular scale), above introduced. It is the basic model chosen to interpret the force-extension curves of force-spectroscopy experiments, including the ones with folding/unfolding transitions. ${ }^{42,49,50}$

The folding/unfolding of the units, here described by the spin variables, is controlled by the mechanical boundary conditions applied to the chain and by the chemical potentials of the external environment. We consider here a chain with the first extremity tethered at the origin of the reference frame. In general, an arbitrary chain described by Eq.(1) can be studied within the Helmholtz ensemble or within the Gibbs ensemble of the statistical mechanics. In the first situation, we fix the second extremity at a given point of the space while, in the second situation, we apply an external force to the last unit. In the following, we briefly describe the statistical mechanics of both ensembles, eventually verifying that the proposed approach yields results, which are coherent with standard thermodynamics.

Within the Helmholtz ensemble we have a specific Hamiltonian given by

$$
H_{H}=H(\vec{q}, \vec{p}, \vec{S} ; \vec{r}, \vec{\mu})
$$

where we introduced $\vec{q}=\left(\vec{r}_{1}, \ldots, \vec{r}_{N-1}\right)$ (we fixed $\vec{r}_{N}=\vec{r}$ ), $\vec{p}=\left(\vec{p}_{1}, \ldots, \vec{p}_{N-1}\right)\left(\right.$ we fixed $\left.\vec{p}_{N}=0\right), \vec{S}=\left(S_{1}, \ldots, S_{N}\right)$, and $\vec{\mu}=\left(\mu_{1}, \ldots, \mu_{N}\right)$. In this scheme, $\vec{r}$ and $\vec{\mu}$ are externally controlled variables and $\vec{q}, \vec{p}$ and $\vec{S}$ are the dynamical variables of the phase space. Therefore, the density probability of the canonical ensemble is given by ${ }^{33,89}$

$$
\rho_{H}(\vec{q}, \vec{p}, \vec{S})=\frac{1}{Z_{H}(\vec{r}, \vec{\mu}, T)} \exp \left[-\frac{H_{H}(\vec{q}, \vec{p}, \vec{S} ; \vec{r}, \vec{\mu})}{k_{B} T}\right],
$$

where the Helmholtz partition function reads

$$
\begin{aligned}
& Z_{H}(\vec{r}, \vec{\mu}, T) \\
& =\sum_{\vec{S} \in\{0,1\}^{N}} \int_{\mathcal{Q}} \int_{\mathcal{Q}} \exp \left[-\frac{H_{H}(\vec{q}, \vec{p}, \vec{S} ; \vec{r}, \vec{\mu})}{k_{B} T}\right] d \vec{q} d \vec{p},
\end{aligned}
$$

and where $\mathcal{Q}=\mathbb{R}^{3(N-1)}$. By means of this probability density, one can determine the average value of any macroscopic variable. In particular, the average value of the force $\vec{f}=\frac{\partial H_{H}}{\partial \vec{r}}$ needed to fix $\vec{r}_{N}=\vec{r}$ and the average value of each spin variable $S_{i}$ can be easily obtained through the introduction of the Helmholtz free energy $\mathcal{F}=-K_{B} T \log Z_{H}$. Indeed, straightforward calculations yield $\langle\vec{f}\rangle=\frac{\partial \mathcal{F}}{\partial \vec{r}}$ and $\langle\vec{S}\rangle=-\frac{\partial \mathcal{F}}{\partial \vec{\mu}}$, which represent two macroscopic or thermodynamic relations. Moreover, the first and second principles for quasi-static transformations can be derived as follows from previous statements. First of all, we define a quasi-static transformation through the time evolution of $\vec{r}(t), \vec{\mu}(t)$ and $T(t)$, which are considered as externally controlled "slow-varying" variables. Under these hypotheses, we can assume that the canonical distribution in Eq.(4) remains valid also in this "weak" out-of-equilibrium regime. Therefore, we can evaluate the rate of change of the internal energy $\mathcal{U}$, defined as the average value of the Helmholtz Hamiltonian

$$
\begin{aligned}
\frac{d \mathcal{U}}{d t}= & \sum_{\vec{S}} \int_{\mathcal{Q}} \int_{\mathcal{Q}} \frac{d}{d t}\left(H_{H} \rho_{H}\right) d \vec{q} d \vec{p} \\
= & \sum_{\vec{S}} \int_{\mathcal{Q}} \int_{\mathcal{Q}}\left(\frac{\partial H_{H}}{\partial \vec{\mu}} \cdot \frac{d \vec{\mu}}{d t}+\frac{\partial H_{H}}{\partial \vec{r}} \cdot \frac{d \vec{r}}{d t}\right) \rho_{H} d \vec{q} d \vec{p} \\
& +\sum_{\vec{S}} \int_{\mathcal{Q}} \int_{\mathcal{Q}} H_{H} \frac{d \rho_{H}}{d t} d \vec{q} d \vec{p} \\
= & -\langle\vec{S}\rangle \cdot \frac{d \vec{\mu}}{d t}+\langle\vec{f}\rangle \cdot \frac{d \vec{r}}{d t}+\sum_{\vec{S}} \int_{\mathcal{Q}} \int_{\mathcal{Q}} H_{H} \frac{d \rho_{H}}{d t} d \vec{q} d \vec{p}
\end{aligned}
$$

where all the sums on $\vec{S}$ are performed over $\{0,1\}^{N}$. The first two terms represent the chemical and the mechanical work per unit of time, respectively, done on the system, while the third one describes the rate of heat entering the system. The latter can be further elaborated by defining $\eta=\frac{\mathcal{F}-H_{H}}{k_{B} T}$ and $\rho_{H}=e^{\eta}$. Since $\sum_{\vec{S}} \int_{\mathcal{Q}} \int_{\mathcal{Q}} e^{\eta} d \vec{q} d \vec{p}=1$, the derivative with respect to the time gives $\sum_{\vec{S}} \int_{\mathcal{Q}} \int_{\mathcal{Q}} e^{\eta} \dot{\eta} d \vec{q} d \vec{p}=0$, and since $\mathcal{F}$ is independent of $\vec{S}, \vec{q}$ and $\vec{p}$, we can also write that $\sum_{\vec{S}} \int_{\mathcal{Q}} \int_{\mathcal{Q}} \mathcal{F} e^{\eta} \dot{\eta} d \vec{q} d \vec{p}=0$. The last term in Eq.(6) can 
be therefore rewritten as

$$
\begin{aligned}
\sum_{\vec{S}} \int_{\mathcal{Q}} \int_{\mathcal{Q}} H_{H} \frac{d \rho_{H}}{d t} d \vec{q} d \vec{p} & =\sum_{\vec{S}} \int_{\mathcal{Q}} \int_{\mathcal{Q}} H_{H} e^{\eta} \dot{\eta} d \vec{q} d \vec{p} \\
& =\sum_{\vec{S}} \int_{\mathcal{Q}} \int_{\mathcal{Q}}\left(H_{H}-\mathcal{F}\right) e^{\eta} \dot{\eta} d \vec{q} d \vec{p} .
\end{aligned}
$$

Now, since $H_{H}-\mathcal{F}=-k_{B} T \eta$, we also have

$$
\sum_{\vec{S}} \int_{\mathcal{Q}} \int_{\mathcal{Q}} H_{H} \frac{d \rho_{H}}{d t} d \vec{q} d \vec{p}=-k_{B} T \sum_{\vec{S}} \int_{\mathcal{Q}} \int_{\mathcal{Q}} e^{\eta} \eta \dot{\eta} d \vec{q} d \vec{p} .
$$

This expression can be easily interpreted by considering the average value of the variable $\eta$ given by $\langle\eta\rangle=$ $\sum_{\vec{S}} \int_{\mathcal{Q}} \int_{\mathcal{Q}} e^{\eta} \eta d \vec{q} d \vec{p}$ and its time derivatives

$$
\frac{d\langle\eta\rangle}{d t}=\sum_{\vec{S}} \int_{\mathcal{Q}} \int_{\mathcal{Q}} e^{\eta} \dot{\eta} d \vec{q} d \vec{p}+\sum_{\vec{S}} \int_{\mathcal{Q}} \int_{\mathcal{Q}} e^{\eta} \eta \dot{\eta} d \vec{q} d \vec{p}
$$

Since the first integral is zero for previous calculations, we eventually obtain

$$
\sum_{\vec{S}} \int_{\mathcal{Q}} \int_{\mathcal{Q}} H_{H} \frac{d \rho_{H}}{d t} d \vec{q} d \vec{p}=-k_{B} T \frac{d\langle\eta\rangle}{d t}=T \frac{d \mathcal{S}}{d t},
$$

where we introduced the entropy of the system as

$$
\mathcal{S}=-k_{B}\left\langle\log \rho_{H}\right\rangle=-k_{B} \sum_{\vec{S}} \int_{\mathcal{Q}} \int_{\mathcal{Q}} \rho_{H} \log \rho_{H} d \vec{q} d \vec{p}
$$

The first and the second principles of the thermodynamics for quasi-static transformations can be finally summed up through the balance equation

$$
\frac{d \mathcal{U}}{d t}=-\langle\vec{S}\rangle \cdot \frac{d \vec{\mu}}{d t}+\langle\vec{f}\rangle \cdot \frac{d \vec{r}}{d t}+T \frac{d \mathcal{S}}{d t} .
$$

Hence, we proved that the macroscopic thermodynamics is perfectly coherent with the approach based on the spin variables, introduced to analyze systems with conformational transitions. In addition, the average value of the relation $H_{H}-\mathcal{F}=-k_{B} T \eta$ yields $\mathcal{F}=\mathcal{U}-T \mathcal{S}$, which corresponds to the macroscopic definition of Helmholtz free energy. By differentiating with respect to the time, we also have

$$
\frac{d \mathcal{F}}{d t}=-\langle\vec{S}\rangle \cdot \frac{d \vec{\mu}}{d t}+\langle\vec{f}\rangle \cdot \frac{d \vec{r}}{d t}-\mathcal{S} \frac{d T}{d t}
$$

from which we can deduce the two already introduced equations $\langle\vec{f}\rangle=\frac{\partial \mathcal{F}}{\partial \vec{r}}$ and $\langle\vec{S}\rangle=-\frac{\partial \mathcal{F}}{\partial \vec{\mu}}$ and the important relation $\mathcal{S}=-\frac{\partial \mathcal{F}}{\partial T}$, giving the entropy in terms of the Helmholtz free energy.

Concerning the Gibbs ensemble, we can introduce the following extended Hamiltonian

$$
H_{G}=H(\vec{q}, \vec{p}, \vec{S} ; \vec{\mu})-\vec{f} \cdot \vec{r}_{N},
$$

where the second term represents the energy associated to the external force applied to the last unit of the chain.
Here, we introduced $\vec{q}=\left(\vec{r}_{1}, \ldots, \vec{r}_{N}\right), \vec{p}=\left(\vec{p}_{1}, \ldots, \vec{p}_{N}\right), \vec{S}=$ $\left(S_{1}, \ldots, S_{N}\right)$, and $\vec{\mu}=\left(\mu_{1}, \ldots, \mu_{N}\right)$. In this scheme, $\vec{f}$ and $\vec{\mu}$ are externally controlled variables and $\vec{q}, \vec{p}$ and $\vec{S}$ are the dynamical variables of the phase space. Therefore, the density probability of the canonical ensemble is given by $^{33,89}$

$\rho_{G}(\vec{q}, \vec{p}, \vec{S})=\frac{1}{Z_{G}(\vec{f}, \vec{\mu}, T)} \exp \left[-\frac{H_{G}(\vec{q}, \vec{p}, \vec{S} ; \vec{f}, \vec{\mu})}{k_{B} T}\right]$,

where the Gibbs partition function reads

$$
\begin{aligned}
& Z_{G}(\vec{f}, \vec{\mu}, T) \\
= & \sum_{\vec{S} \in\{0,1\}^{N}} \int_{\mathcal{P}} \int_{\mathcal{P}} \exp \left[-\frac{H_{G}(\vec{q}, \vec{p}, \vec{S} ; \vec{f}, \vec{\mu})}{k_{B} T}\right] d \vec{q} d \vec{p},
\end{aligned}
$$

and where $\mathcal{P}=\mathbb{R}^{3 N}$. As before, the most important expected values can be evaluated through the introduction of the Gibbs free energy $\mathcal{G}=-k_{B} T \log Z_{G}$. Simple calculations yield indeed $\langle\vec{r}\rangle=-\frac{\partial \mathcal{G}}{\partial \vec{f}}$ (where $\vec{r}=\vec{r}_{N}$ ) and $\langle\vec{S}\rangle=-\frac{\partial \mathcal{G}}{\partial \vec{\mu}}$, which represent two classical thermodynamic relations. Also in this isotensional case, we can introduce a quasi-static transformation through the time evolution of $\vec{f}(t), \vec{\mu}(t)$ and $T(t)$, which are, as before, externally controlled "slow-varying" variables. We can assume that the canonical distribution in Eq.(15) remains valid and we can evaluate the rate of change of the average value of the Gibbs Hamiltonian, which is the so-called enthalpy $\mathcal{H}$

$$
\begin{aligned}
\frac{d \mathcal{H}}{d t}= & \sum_{\vec{S}} \int_{\mathcal{P}} \int_{\mathcal{P}} \frac{d}{d t}\left(H_{G} \rho_{G}\right) d \vec{q} d \vec{p} \\
= & \sum_{\vec{S}} \int_{\mathcal{P}} \int_{\mathcal{P}}\left(\frac{\partial H_{G}}{\partial \vec{\mu}} \cdot \frac{d \vec{\mu}}{d t}+\frac{\partial H_{G}}{\partial \vec{f}} \cdot \frac{d \vec{f}}{d t}\right) \rho_{G} d \vec{q} d \vec{p} \\
& +\sum_{\vec{S}} \int_{\mathcal{P}} \int_{\mathcal{P}} H_{G} \frac{d \rho_{G}}{d t} d \vec{q} d \vec{p} \\
= & -\langle\vec{S}\rangle \cdot \frac{d \vec{\mu}}{d t}-\langle\vec{r}\rangle \cdot \frac{d \vec{f}}{d t}+\sum_{\vec{S}} \int_{\mathcal{P}} \int_{\mathcal{P}} H_{G} \frac{d \rho_{G}}{d t} d \vec{q} d \vec{p} .
\end{aligned}
$$

While the first two terms represent the power (chemical and mechanical) supplied to the system, the third term is the rate of heat transferred to the system. A calculation similar to the one developed for the Helmholtz ensemble allows us to rewrite this last term as follows

$$
\frac{d \mathcal{H}}{d t}=-\langle\vec{S}\rangle \cdot \frac{d \vec{\mu}}{d t}-\langle\vec{r}\rangle \cdot \frac{d \vec{f}}{d t}+T \frac{d \mathcal{S}}{d t},
$$

where the entropy for the Gibbs ensemble is given by

$$
\mathcal{S}=-k_{B}\left\langle\log \rho_{G}\right\rangle=-k_{B} \sum_{\vec{S}} \int_{\mathcal{P}} \int_{\mathcal{P}} \rho_{G} \log \rho_{G} d \vec{q} d \vec{p} .
$$

We can straightforwardly prove the thermodynamic relation $\mathcal{G}=\mathcal{H}-T \mathcal{S}$ and we obtain the rate of change of 
the Gibbs free energy as

$$
\frac{d \mathcal{G}}{d t}=-\langle\vec{S}\rangle \cdot \frac{d \vec{\mu}}{d t}-\langle\vec{r}\rangle \cdot \frac{d \vec{f}}{d t}-\mathcal{S} \frac{d T}{d t} .
$$

The last energy balance immediately delivers the relations $\langle\vec{r}\rangle=-\frac{\partial \mathcal{G}}{\partial \vec{f}}$ and $\langle\vec{S}\rangle=-\frac{\partial \mathcal{G}}{\partial \vec{\mu}}$, previously demonstrated, and the result $\mathcal{S}=-\frac{\partial \mathcal{G}}{\partial T}$, corresponding to the macroscopic definition of entropy.

The two schemes here outlined represent a complete description of the thermo-mechanical response of a macromolecular chain undergoing conformational transitions under isometric or isotensional boundary conditions. We proved that the introduction of additional "spin" variables to describe the folding or unfolding of the units leaves unaltered the general results of the thermodynamics, thus being well-grounded and promising for applications. As an example, the application of this methodology to the bistable freely jointed chain can be found in the recent literature. ${ }^{76}$ It is important to remark that the thermo-elastic response may be different for the two introduced ensembles if we consider chains composed of a small number $N$ of units. ${ }^{33}$ On the other hand, when the thermodynamic limit is attained (ideally for $N \rightarrow \infty$ ), the two ensembles become equivalent and they exhibit the same physical response. ${ }^{37,52-55}$ This equivalence property is valid for non-branched single chains without confinements, i.e. freely fluctuating in the whole space. ${ }^{54}$ Therefore, in our analysis, the only constraints consist in the punctual boundary conditions defining the Helmholtz and the Gibbs ensembles. However, it is noteworthy to mention that some particular cases on non-equivalence between dual canonical ensembles have been recently discussed for confined polymer chains. ${ }^{90-92}$

\section{TWO-STATE FREELY JOINTED CHAIN WITH EXTENSIBLE UNITS: THE GIBBS ENSEMBLE}

We take into consideration a two-state freely jointed chain composed of $N$ domains. Here, instead of considering the units with an infinite elastic stiffness as in Ref.76, we try to consider a finite elasticity for the two-state domains. As previosly anticipated, the potential energy function of real domains is represented by a function with two minima, corresponding to the folded and unfolded configurations of the domain (dashed black curve in Fig.2). We introduce two quadratic potential curves, approximating the wells of this system, and a spin variable $S_{i}$ for each unit, useful to identify the potential well explored during the system evolution (see again Fig.2). The switching of $S_{i}$ is controlled by the boundary conditions applied to the system, here defined by the force $\vec{f}$ (Gibbs ensemble). A first validation of the spin approach to describe multistable potential energies has been performed in Ref.76, where we directly

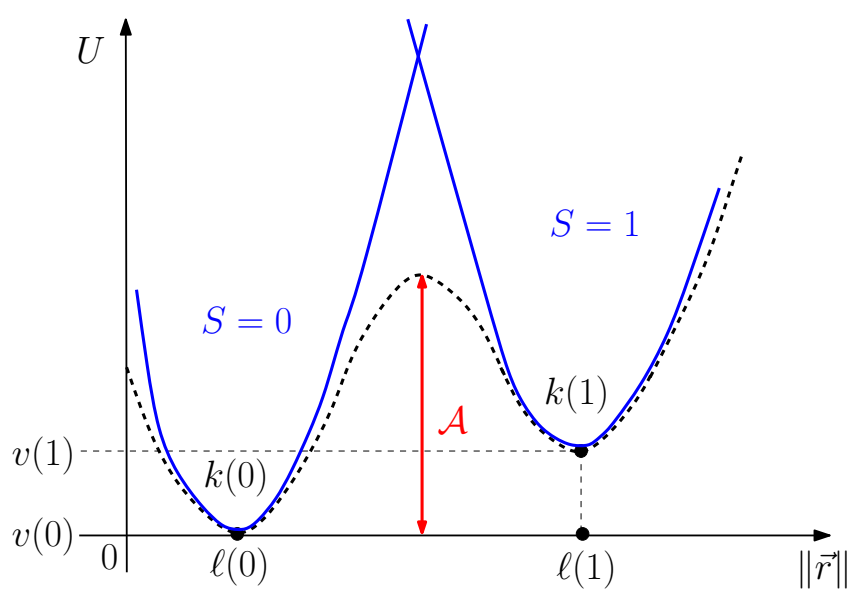

FIG. 2. Potential energy of a single unit of the two-state freely jointed chain with extensible links (dashed black curve). The potential wells are approximated through two parabolic profiles identified by $S=0$ and 1 (solid blues curves).

compared a real two-state system (dashed black curve in Fig.2) with the approximation given by two parabolic profiles governed by the spin variables (solid blue curves in Fig.2). By means of Monte Carlo simulations and a theoretical comparison (at thermodynamic equilibrium), we verified that this method offers a very high accuracy if $\mathcal{A} \geq v(1)-v(0) \gg k_{B} T$, where $\mathcal{A}$ is the energy barrier between the states and $v(1)-v(0)$ represents the energy jump (see Fig.2).

We start the analysis of the Gibbs ensemble by writing the extended Hamiltonian of the system, as follows

$$
\begin{aligned}
H_{G}= & \sum_{i=1}^{N} \frac{\vec{p}_{i} \cdot \vec{p}_{i}}{2 M}+\sum_{i=1}^{N} v\left(S_{i}\right)-\mu \sum_{i=1}^{N} S_{i} \\
& +\sum_{i=1}^{N} \frac{1}{2} k\left(S_{i}\right)\left[\left\|\vec{r}_{i}-\vec{r}_{i-1}\right\|-\ell\left(S_{i}\right)\right]^{2}-\vec{f} \cdot \vec{r}_{N}
\end{aligned}
$$

where all variables are defined in Section II. Moreover, the quantities $v\left(S_{i}\right), k\left(S_{i}\right)$ and $\ell\left(S_{i}\right)$ stand for the basal energy, the elastic stiffness and the equilibrium length of the potential wells, respectively (see Fig.2 for details). From the physical point of view, the basal energy of a potential well represents its minimum of energy, namely the energetic level of the unit in its stable (or metastable) state. The sequence of different basal energies for a unit defines therefore the multi-basin energy landscape of the unit itself, i.e. the most important description of its chemical and physical behavior. Moreover, we introduce $\vec{q}=\left(\vec{r}_{1}, \ldots, \vec{r}_{N}\right)$ as the generalized vector containing all positions, $\vec{p}=\left(\vec{p}_{1}, \ldots, \vec{p}_{N}\right)$ as the generalized vector containing all momenta, and $\vec{S}=\left(S_{1}, \ldots, S_{N}\right)$ as the vector of all spin variables. For the sake of simplicity, we assume that all chemical potentials $\mu_{i}$ take the same value $\mu$.

Since the dynamical variables are $\vec{q}, \vec{p}$ and $\vec{S}$, the par- 
tition function is given by

$$
\begin{aligned}
Z_{G}(\vec{f})= & \sum_{\vec{S} \in\{0,1\}^{N}} \int_{\mathcal{P}} \int_{\mathcal{P}} \exp \left[-\frac{H_{G}(\vec{p}, \vec{q}, \vec{S})}{K_{B} T}\right] \mathrm{d} \vec{p} \mathrm{~d} \vec{q} \\
= & \sum_{\vec{S} \in\{0,1\}^{N}}\left[\int_{\mathbb{R}^{3}} \exp \left(-\frac{1}{2 M K_{B} T} \vec{p} \cdot \vec{p}\right) \mathrm{d} \vec{p}\right]^{N} \\
& \times \int_{\mathbb{R}^{3}} \ldots \int_{\mathbb{R}^{3}} \exp \left[-\sum_{i=1}^{N} \frac{v\left(S_{i}\right)-\mu S_{i}}{K_{B} T}+\frac{\vec{f} \cdot \vec{r}_{N}}{K_{B} T}\right. \\
& \left.-\sum_{i=1}^{N} \frac{k\left(S_{i}\right)}{2 K_{B} T}\left[\left\|\vec{r}_{i}-\vec{r}_{i-1}\right\|-\ell\left(S_{i}\right)\right]^{2}\right] \mathrm{d} \vec{r}_{1} \ldots \mathrm{d} \vec{r}_{N},
\end{aligned}
$$

where $\mathcal{P}=\mathbb{R}^{3 N}$. To begin the simplification of this expression, we can firstly calculate the integral over the linear momentum $\vec{p}$. Incidentally, we observe that the kinetic energy in Eq.(21) simply produces a non-influential multiplicative constant in $Z_{G}(\vec{f})$. We considered this term in Eq.(21) just to have a full Hamiltonian description of the system, coherently with Section II. Then, we perform the change of variables $\vec{\xi}_{1}=\vec{r}_{1}-\vec{r}_{0}, \ldots$ $\vec{\xi}_{N}=\vec{r}_{N}-\vec{r}_{N-1}$, from which we have that $d \vec{r}_{1} \ldots d \vec{r}_{N}=$ $d \vec{\xi}_{1} \ldots d \vec{\xi}_{N}$. So, by fixing $\vec{r}_{0}=0$, we obtain $\vec{r}_{N}=\sum_{i=1}^{N} \vec{\xi}_{i}$, and the partition function becomes

$$
\begin{aligned}
Z_{G}(\vec{f})= & \left(2 \pi M K_{B} T\right)^{3 N / 2}\left\{\sum _ { S \in \{ 0 , 1 \} } \int _ { \mathbb { R } ^ { 3 } } \operatorname { e x p } \left[-\frac{v(S)-\mu S}{K_{B} T}\right.\right. \\
& \left.\left.-\frac{k(S)}{2 K_{B} T}[\|\vec{\xi}\|-\ell(S)]^{2}+\frac{\vec{f} \cdot \vec{\xi}}{K_{B} T}\right] \mathrm{~d} \vec{\xi}\right\}^{N} \cdot
\end{aligned}
$$

It is important to remark that within the Gibbs ensemble, the elements of the chain do not interact and this point leads to a factorized partition function, namely in the form of a power with exponent $N$. We can further simplify the calculation by means of the spherical coordinates $\vec{\xi}=(\xi \cos \varphi \sin \vartheta, \xi \sin \varphi \sin \vartheta, \xi \cos \vartheta)$. Because of the spherical symmetry of the problem, we set $\vec{f}=(0,0, f)$. Since $d \vec{\xi}=\xi^{2} \sin \vartheta d \xi d \varphi d \vartheta,\|\vec{\xi}\|=\xi$ and $\vec{f} \cdot \vec{\xi}=f \xi \cos \vartheta$, we get the following simpler form of the partition function

$$
\begin{aligned}
& Z_{G}(f)=\left(2 \pi M K_{B} T\right)^{3 N / 2}(4 \pi)^{N}\left[\sum_{S \in\{0,1\}} \exp \left[-\frac{v(S)-\mu S}{K_{B} T}\right]\right. \\
& \left.\int_{0}^{+\infty} \exp \left[-\frac{k(S)}{2 K_{B} T}[\xi-\ell(S)]^{2}\right] \frac{\sinh \left(\frac{f \xi}{K_{B} T}\right)}{\frac{f \xi}{K_{B} T}} \xi^{2} \mathrm{~d} \xi\right]^{N} .
\end{aligned}
$$

Now, in order to specify the shape of the potential wells represented in Fig.2, we consider $\ell(0)=\ell, \ell(1)=$ $\chi \ell, v(0)=0, v(1)=\Delta E$ and $k(0)=k(1)=k$. From now on, we neglect the non influential multiplicative constant within the partition function. Hence, we obtain

$$
\begin{aligned}
Z_{G}(f)= & \left\{\int_{0}^{+\infty} e^{-\frac{k}{2 K_{B} T}[\xi-\ell]^{2}} \frac{\sinh \left(\frac{f \xi}{K_{B} T}\right)}{\frac{f \xi}{K_{B} T}} \xi^{2} \mathrm{~d} \xi\right. \\
& \left.+\phi \int_{0}^{+\infty} e^{-\frac{k}{2 K_{B} T}[\xi-\chi \ell]^{2}} \frac{\sinh \left(\frac{f \xi}{K_{B} T}\right)}{\frac{f \xi}{K_{B} T}} \xi^{2} \mathrm{~d} \xi\right\}^{N},
\end{aligned}
$$

where $\phi=\exp \left(-\frac{\Delta E-\mu}{K_{B} T}\right)$. We note that the chemical potential $\mu$ acts on the system by simply shifting the jump energy $\Delta E$. The exact evaluation of previous integrals can be easily done through the known result ${ }^{93}$

$$
\begin{aligned}
& \int_{0}^{+\infty} \exp \left(-\alpha x^{2}\right) \exp (\beta x) x \mathrm{~d} x \\
& =\frac{1}{2 \alpha}+\frac{\beta}{4 \alpha} \sqrt{\frac{\pi}{\alpha}} \exp \left(\frac{\beta^{2}}{4 \alpha}\right)\left[1+\operatorname{erf}\left(\frac{\beta}{2 \sqrt{\alpha}}\right)\right],
\end{aligned}
$$

where, as usual, the error function $\operatorname{erf}(z)$ is defined as $\operatorname{erf}(z)=\frac{2}{\sqrt{\pi}} \int_{0}^{z} \exp \left(-t^{2}\right) \mathrm{d} t{ }^{94}$ Indeed, if we rewrite Eq.(25) in the form

$$
Z_{G}(f)=\left\{I_{1}+\phi I_{2}\right\}^{N},
$$

we have

$$
\begin{aligned}
I_{1}= & \frac{K_{B} T}{4 f} \sqrt{\frac{2 \pi K_{B} T}{k}} \exp \left(\frac{f^{2}}{2 k K_{B} T}\right) \\
& \times\left[\left(\ell+\frac{f}{k}\right) \exp \left(\frac{\ell f}{K_{B} T}\right)\left[1+\operatorname{erf}\left(\frac{k \ell+f}{\sqrt{2 k K_{B} T}}\right)\right]\right. \\
& \left.-\left(\ell-\frac{f}{k}\right) \exp \left(-\frac{\ell f}{K_{B} T}\right)\left[1+\operatorname{erf}\left(\frac{k \ell-f}{\sqrt{2 k K_{B} T}}\right)\right]\right],
\end{aligned}
$$

and $I_{2}$ can be simply obtained by replacing $\ell$ with $\chi \ell$ in $I_{1}$ (see Eq.(25)). Finally, Eq.(27) represents the exact evaluation of the partition function for our system within the Gibbs ensemble.

Now, we are interested in extensible links with relatively high values of the elastic constant $k$. Therefore, we can use the asymptotic expression $\operatorname{erf}(x) \simeq$ $1-\frac{1}{\sqrt{\pi} x} \exp \left(-x^{2}\right)$, holding for large values of $x \cdot{ }^{93,94}$ If we consider $f / k \ll \ell$, we have that the arguments of the error function are proportional to $\sqrt{k}$ and the terms $\operatorname{erf}\left(\frac{k \ell \pm f}{\sqrt{2 k K_{B} T}}\right)$ converge exponentially to 1 with relatively high values of $k$. Typical values of the elastic constants obtained from experiments as well as ab initio calculations can be found in literature and are perfectly compatible with such approximations. ${ }^{95-98}$ We define the variable $y=\frac{\ell f}{K_{B} T}$ (dimensionless force) and the parameter $\alpha=\frac{K_{B} T}{k \ell^{2}}$ (ratio between thermal energy and elastic one). Hence, the partition function is given by the following approximation

$$
\begin{aligned}
& Z_{G}(f)=\exp \left(\frac{N \alpha y^{2}}{2}\right) \\
& \times\left(\frac{\sinh y}{y}+\alpha \cosh y+\phi \chi \frac{\sinh \chi y}{y}+\phi \alpha \cosh \chi y\right)^{N} .
\end{aligned}
$$



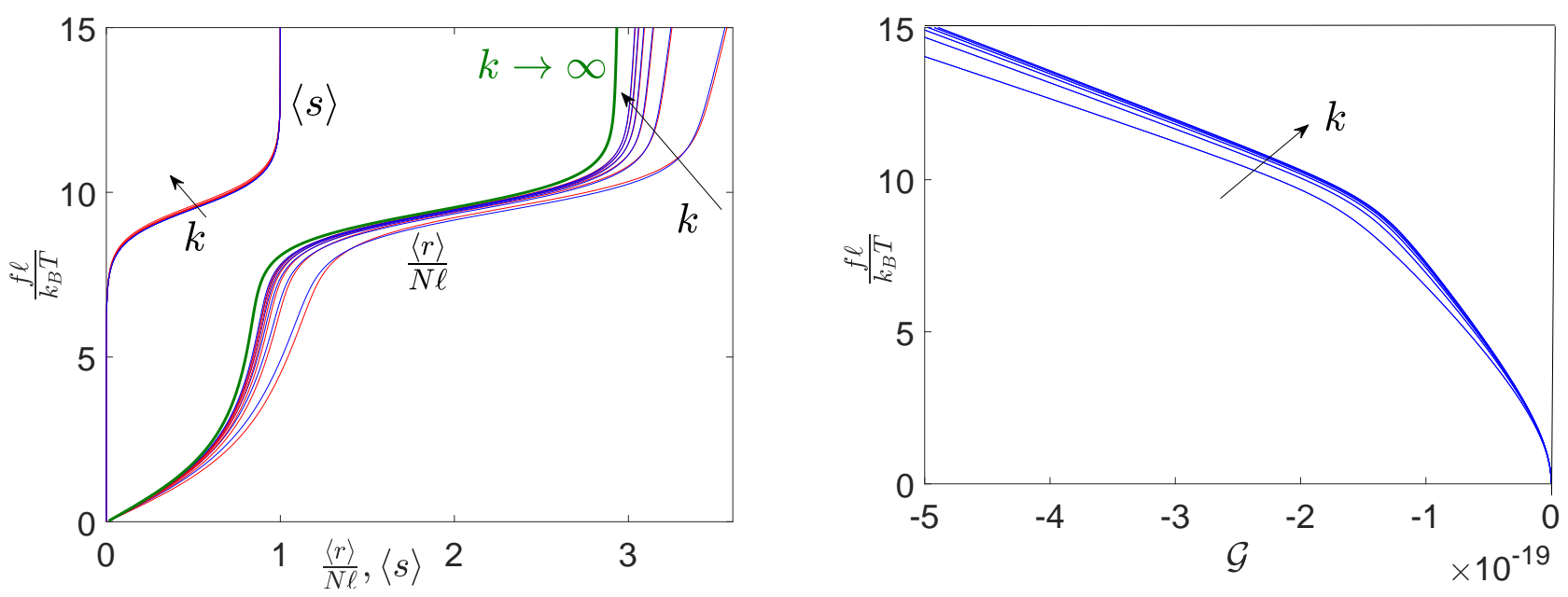

FIG. 3. Force-extension response and average spin (left panel) and Gibbs free energy (right panel) for a system with variable extensibility $k=0.4 j \mathrm{~N} / \mathrm{m}, \forall j=1, \ldots, 6$. The Gibbs free energy $\mathcal{G}$ is represented in Joule. The calculation of the force-extension response and the average spin is based on the exact result in Eq.(27) (red curves) and on the approximation in Eq.(30) (blue curves). In left panel, we also added the response (green curve) for the system without extensibility $(k \rightarrow \infty)$, studied through Eq.(31). We adopted the parameters $N=5, \ell=0.5 \times 10^{-9} \mathrm{~m}, T=300 \mathrm{~K}, \chi=3, \Delta E=20 k_{B} T=8.28 \times 10^{-20} \mathrm{~J}$ and $\mu=0$.

Finally, if we suppose to have $\alpha \ll 1$, we obtain the further approximation

$$
Z_{G}(f)=\left(\frac{\sinh y}{y}+\phi \chi \frac{\sinh \chi y}{y}\right)^{N} \exp \left(\frac{N \alpha y^{2}}{2}\right),
$$

which will be used throughout all this paper. The exponential term depending on $\alpha$ is responsible for the elasticity of the chain. The same result without bistability (i.e. with $\phi=0)$, has been discussed in recent literature. ${ }^{99,100}$ Moreover, the case with bistability but without extensibility of the units is considered in Ref.76. In this situation the partition function simply reduces to

$$
\lim _{\alpha \rightarrow 0} Z_{G}(f)=\left(\frac{\sinh y}{y}+\phi \chi \frac{\sinh \chi y}{y}\right)^{N} .
$$

As discussed in Section II, the macroscopic behavior of this system is described by the Gibbs free energy $\mathcal{G}=$ $-k_{B} T \log Z_{G}$. The force-extension response for the twostate freely jointed chain with extensible elements under isotensional condition can be found through

$$
\langle r\rangle=-\frac{\partial \mathcal{G}}{\partial f} .
$$

On the other hand, if we define the average value of the spin variables as $\langle s\rangle=\frac{1}{N}\left\langle\sum_{i=1}^{N} S_{i}\right\rangle$, it is not difficult to prove that

$$
N\langle s\rangle=\frac{\partial \mathcal{G}}{\partial \Delta E}=-\frac{\partial \mathcal{G}}{\partial \mu} .
$$

The explicit application of Eq.(32) to Eq.(30) yields

$$
\langle r\rangle=N \ell\left[\frac{\mathcal{L}(y)+\chi^{2} \phi \mathcal{L}(\chi y) \frac{\sinh \chi y}{\sinh y}}{1+\chi \phi \frac{\sinh \chi y}{\sinh y}}+\alpha y\right],
$$

where $\mathcal{L}(y)=\operatorname{coth} y-\frac{1}{y}$ is the Langevin function. On the other hand, by applying Eq.(33) to Eq.(30), we get

$$
\langle s\rangle=\frac{\chi \phi \frac{\sinh \chi y}{\sinh y}}{1+\chi \phi \frac{\sinh \chi y}{\sinh y}},
$$

which does not depend on $\alpha$. By combining Eqs.(34) and (35), we can find the relationship

$$
\langle r\rangle=N \ell[(1-\langle s\rangle) \mathcal{L}(y)+\langle s\rangle \chi \mathcal{L}(\chi y)+\alpha y],
$$

stating that the average extension of the extensible twostate system is given by a combination of the responses of two FJC models with length $\ell$ and $\chi \ell$, and by a linear term taking into account the elasticity of the system. This result is similar in spirit to that presented in Ref.99.

An example of application of Eqs.(32) and (33) is given in Fig.3, where force-extension curves, spin variables and Gibbs free energies are plotted for several values of $k=0.4 j \mathrm{~N} / \mathrm{m}, \forall j=1, \ldots, 6$. We remark that real macromolecular elastic constants are similar to or larger than the values adopted in this analysis. ${ }^{95-98}$ In the first panel, we compare the systems with extensibility, described by the exact Eq.(27) or by the approximated Eq.(30), and another without extensibility $(k \rightarrow \infty)$ described by Eq.(31). First of all, we remark the very good agreement between Eqs.(27) and Eq.(30) for the systems with extensibility (red and blues curves, respectively). This proves the acceptability of the proposed approximation. Moreover, it is interesting to note the different asymptotic behavior of systems with finite and infinite elastic constants. While the stiff system exhibits a vertical asymptote for large forces (green curve), the elastic ones shows an oblique asymptote (red and blues curves), which slope represents the effective stiffness of the chain. 
In the force-extension curves, we observe a force plateau corresponding to $f^{*}=\frac{\Delta E}{(\chi-1) \ell}$ (in the case of $\mu=0$ ). Similarly, in the spin behavior we identify a transition from $\langle s\rangle=0$ to $\langle s\rangle=1$ corresponding to the same force $f^{*}$. We can explain these curves by means of a cooperative process, which generates the transition of all units at the same value of force $f^{*}$. Notably, the value of this plateau force does not depend on the spring constant, nor on the temperature. Such a result is readily interpreted in the framework of the Bell expression, originally derived in the context of the adhesion of cells. ${ }^{101}$ While the spin variable average remains unaltered by the elastic constant, the force-extension response is sensibly modified, showing a variable slope in the regions before and after the central plateau. The origin of the transition in the force-extension curve and in the spin behavior can be recognized in the Gibbs free energy, which shows a slope change corresponding to the same force $f^{*}$. The knowledge of chain behavior under the isotensional or Gibbs ensemble is the starting point for the understanding of the isometric ensemble, as discussed in the next Section.

\section{TWO-STATE FREELY JOINTED CHAIN WITH EXTENSIBLE UNITS: THE HELMHOLTZ ENSEMBLE}

We consider now the isometric or Helmholtz ensemble, where the last unit of the chain is considered tethered at a given point $\vec{r}$ of the space. In this case, we can therefore write the Hamiltonian of the system in the form

$$
\begin{aligned}
H_{H}= & \sum_{i=1}^{N-1} \frac{\vec{p}_{i} \cdot \vec{p}_{i}}{2 M}+\sum_{i=1}^{N} v\left(S_{i}\right)-\mu \sum_{i=1}^{N} S_{i} \\
& +\sum_{i=1}^{N} \frac{1}{2} k\left(S_{i}\right)\left[\left\|\vec{r}_{i}-\vec{r}_{i-1}\right\|-\ell\left(S_{i}\right)\right]^{2},
\end{aligned}
$$

where $\vec{r}_{N}=\vec{r}$ is fixed. Consequently, we also considered $\vec{p}_{N}=0$. In this situation, we introduce $\vec{q}=\left(\vec{r}_{1}, \ldots, \vec{r}_{N-1}\right)$ as the generalized vector containing all positions, $\vec{p}=$ $\left(\vec{p}_{1}, \ldots, \vec{p}_{N-1}\right)$ as the generalized vector containing all momenta, and $\vec{S}=\left(S_{1}, \ldots, S_{N}\right)$ as the vector of all spin variables. As before, we assume that all chemical potentials $\mu_{i}$ take the same value $\mu$. The partition function can be written in terms of these dynamic variables

$$
\begin{aligned}
Z_{H}(\vec{r}) & =\sum_{\vec{S} \in\{0,1\}^{N}} \int_{\mathcal{Q}} \int_{\mathcal{Q}} \exp \left[-\frac{H_{H}(\vec{p}, \vec{q}, \vec{S})}{K_{B} T}\right] \mathrm{d} \vec{p} \mathrm{~d} \vec{q} \\
& =\sum_{\vec{S} \in\{0,1\}^{N}}\left[\int_{\mathbb{R}^{3}} \exp \left(-\frac{1}{2 M K_{B} T} \vec{p} \cdot \vec{p}\right) \mathrm{d} \vec{p}\right]^{N-1} \\
& \times \int_{\mathbb{R}^{3}} \ldots \int_{\mathbb{R}^{3}} \exp \left[-\sum_{i=1}^{N} \frac{v\left(S_{i}\right)-\mu S_{i}}{K_{B} T}\right. \\
& \left.-\sum_{i=1}^{N} \frac{k\left(S_{i}\right)}{2 K_{B} T}\left[\left\|\vec{r}_{i}-\vec{r}_{i-1}\right\|-\ell\left(S_{i}\right)\right]^{2}\right] \mathrm{d} \vec{r}_{1} \ldots \mathrm{d} \vec{r}_{N-1},
\end{aligned}
$$

where $\mathcal{Q}=\mathbb{R}^{3 N-3}$. It is not difficult to realize that the calculation of this partition function is much more complicated than the one obtained for the Gibbs ensemble. Indeed, we can not apply a simple change of variables in order to factorize the multi-dimensional integral. From the physical point of view, this difficulty depends on the fact that the isometric condition induces an effective interaction among the units, fixing the sum of all vectors $\vec{r}_{i}-\vec{r}_{i-1}$ (for $i$ from 1 to $N$ ). This problem will produce a final result which can not be written in the form of a power with exponent $N$. An useful technique to cope with this difficulty is the following. By comparing Eqs.(22) and (38), we deduce that the two partition functions $Z_{G}$ and $Z_{H}$ are related through a three-dimensional bilateral Laplace transform, as follows

$$
Z_{G}(\vec{f})=\int_{\mathbb{R}^{3}} Z_{H}(\vec{r}) \exp \left(\frac{\vec{r} \cdot \vec{f}}{k_{B} T}\right) d \vec{r},
$$

where, as usual, we neglect the non-influential multiplicative constants. Moreover, by considering the spherical symmetry of the problem, we easily obtain the inverse relationship

$$
Z_{H}(r)=\int_{-\infty}^{+\infty} Z_{G}(i \eta) \frac{\eta}{r} \sin \frac{\eta r}{k_{B} T} d \eta
$$

where we have introduced the analytic continuation of the function $Z_{G}(r)$ to the imaginary axis, leading to $Z_{G}(i \eta)$. By substituting Eq.(30) in Eq.(40), we get the important integral expression

$$
\begin{aligned}
Z_{H}(r)= & \int_{-\infty}^{+\infty}\left\{\frac{\sin y}{y}+\chi \phi \frac{\sin \chi y}{y}\right\}^{N} \\
& \times \exp \left(-N \frac{\alpha y^{2}}{2}\right) \frac{y}{r} \sin \frac{r y}{\ell} d y .
\end{aligned}
$$

The analysis of such an integral for $\alpha=0$ has been performed in recent literature. ${ }^{76}$ Previous investigations considered the case with $\alpha=0$ and $\phi=0$, thus dealing with the classical FJC model under isometric condition. ${ }^{102-105}$ However, the presence of a finite elasticity of the units, quantified by the parameter $\alpha \neq 0$, completely modifies 


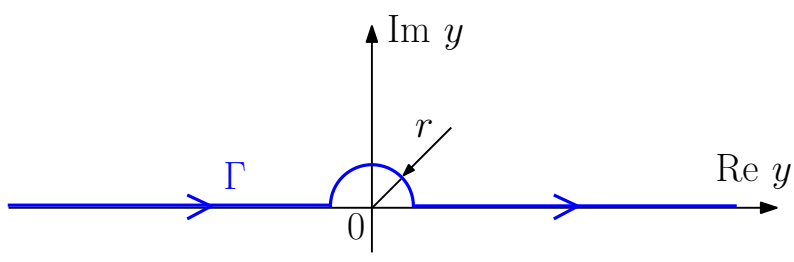

FIG. 4. Definition of the contour $\Gamma$ on the complex plane with an arbitrary radius $r$.

the approach to be used to obtain a closed form expression for $Z_{H}(r)$. Here, we elaborate Eq.(41) in order to get such a closed form expression, useful to better explain the chain behavior within the Helmholtz ensemble. The function to integrate in Eq.(41) is regular on the real axis and analytic (or holomorphic) on a strip $|\operatorname{Im} y|<M$ for an arbitrary $M \in \mathbb{R}$. Then, instead of integrating on the whole real axis, we can use the path $\Gamma$ shown in Fig. 4 . This will be useful to elaborate the partition function integral and to write it in a form with singularities at the origin. Therefore, we can write

$$
\begin{aligned}
Z_{H}(r)= & -i \int_{\Gamma}\left\{\frac{\sin y}{y}+\chi \phi \frac{\sin \chi y}{y}\right\}^{N} \\
& \times \exp \left(-N \frac{\alpha y^{2}}{2}\right) \frac{y}{r} \exp \left(i \frac{r y}{\ell}\right) d y
\end{aligned}
$$

By developing the power in previous expression and by using the expansion

$$
\sin ^{n} x=\frac{1}{(2 i)^{n}} e^{i n x} \sum_{t=0}^{n}\left(\begin{array}{l}
n \\
t
\end{array}\right)(-1)^{t} e^{-2 i t x},
$$

we obtain

$$
\begin{aligned}
Z_{H}(r)= & \frac{1}{2^{N} i^{N+1} r} \sum_{k=0}^{N} \sum_{p=0}^{N-k} \sum_{q=0}^{k}\left(\begin{array}{l}
N \\
k
\end{array}\right)\left(\begin{array}{c}
N-k \\
p
\end{array}\right)\left(\begin{array}{l}
k \\
q
\end{array}\right) \\
& \times(-1)^{p+q}(\chi \phi)^{k} \int_{\Gamma} e^{-i \Lambda y} e^{-N \frac{\alpha y^{2}}{2}} \frac{d y}{y^{N-1}},
\end{aligned}
$$

where we defined

$$
\Lambda=k-N+2 p-\chi k+2 \chi q-\frac{r}{\ell} .
$$

The integral appearing in Eq.(44) is well defined since the path $\Gamma$ excludes the singularity at the origin from the integration.

To perform the calculation of the Helmholtz partition function, we have to determine the value of

$$
I_{m}(a, b)=\int_{\Gamma} e^{i a y} e^{-b y^{2}} \frac{d y}{y^{m}} .
$$

Although this development is an essential part of this paper, for the sake of clarity, the calculations are discussed in Appendices A and B. We first reduce the calculation of Eq.(46) to a simpler integral (Appendix A), and then we solve it through the Hermite elements with negative index (Appendix B). Eventually, the integral $I_{m}(a, b)$ defined in Eq.(46) can be calculated through the expression

$I_{m}(a, b)=\sqrt{\pi}\left\{b^{\frac{m-1}{2}} e^{-\frac{a^{2}}{4 b}}(2 i)^{m} H_{-m}\left(-\frac{a}{2 \sqrt{b}}\right)\right.$
$\left.+\mathbf{1}(-a) \sum_{k=0}^{m-1} \frac{(i a)^{m-1-k}(-4 b)^{k / 2}}{i k !(m-1-k) !} \Gamma\left(\frac{k+1}{2}\right)\left[1+(-1)^{k}\right]\right\}$,

where $\mathbf{1}(x)$ represents the Heaviside step function, defined as $\mathbf{1}(x)=1$ if $x \geq 0$, and $\mathbf{1}(x)=0$ if $x<0$, and $H_{-m}(z)$ represent the Hermite elements with negative index, which are a direct generalization of the classical Hermite polynomials (see Appendix B). To conclude, we can use Eq.(47) to obtain the final form of the Helmholtz partition function

$$
\begin{aligned}
& Z_{H}(r)=-\frac{1}{2 r} \sum_{k=0}^{N} \sum_{p=0}^{N-k} \sum_{q=0}^{k}\left(\begin{array}{c}
N \\
k
\end{array}\right)\left(\begin{array}{c}
N-k \\
p
\end{array}\right)\left(\begin{array}{c}
k \\
q
\end{array}\right)(-1)^{p+q} \\
& \times(\chi \phi)^{k}\left[\sqrt{\pi}\left(\frac{N \alpha}{2}\right)^{\frac{N-2}{2}} e^{-\frac{\Lambda^{2}}{2 N \alpha}} H_{-N+1}\left(\frac{\Lambda}{\sqrt{2 N \alpha}}\right)\right. \\
& \left.+\mathbf{1}(\Lambda) \sum_{h=0}^{\left[\frac{N}{2}-1\right]} \frac{(-1)^{N-1} \pi\left(\frac{\Lambda}{2}\right)^{N-2}}{h !(N-2-2 h) !}\left(\frac{N \alpha}{2 \Lambda^{2}}\right)^{h}\right]
\end{aligned}
$$

where $[x]$ represents the floor function giving the greatest integer that is less than or equal to $x$, and $\Lambda$ depends on $k, p$ and $q$ through Eq.(45). This result is the most important achievement of this paper and allows us to determine the complete response of the two-state freely jointed chain with extensible lengths in terms of forceextension curve and average value of the spin variables. Interestingly enough, Eq.(48) represents a closed form expression since all sums are performed over finite ranges and the Hermite elements are known recursively, as discussed in Appendix B. As a check of the procedure, we can see that if $\alpha=0$ (i.e., infinite stiffness) the first term within the brackets vanishes and the second one is given only by the addend with $h=0$. Hence, the partition function simplifies to give

$$
\begin{aligned}
\lim _{\alpha \rightarrow 0} Z_{H}(r)= & \frac{\pi}{2^{(N-1)}(N-2) ! r} \sum_{k=0}^{N} \sum_{p=0}^{N-k} \sum_{q=0}^{k}\left(\begin{array}{c}
N \\
k
\end{array}\right)\left(\begin{array}{c}
N-k \\
p
\end{array}\right) \\
& \times\left(\begin{array}{l}
k \\
q
\end{array}\right)(-1)^{p+q}(\chi \phi)^{k}(-\Lambda)^{N-2} \mathbf{1}(\Lambda),
\end{aligned}
$$

in perfect agreement with Ref.76. In any case, we remark that Eqs.(48) and (49) can not be written as a power with exponent $N$. It means that, within the Helmholtz ensemble, there is an effective interaction among the elements induced by the isometric boundary conditions. However, this interaction is not explicitly written in the Hamiltonian of the system (as, e.g., in the Ising model) but comes from the prescribed positions of the two chain extremities.

All thermodynamic properties can be evaluated through the Helmholtz free energy $\mathcal{F}=-k_{B} T \log Z_{H}$ 

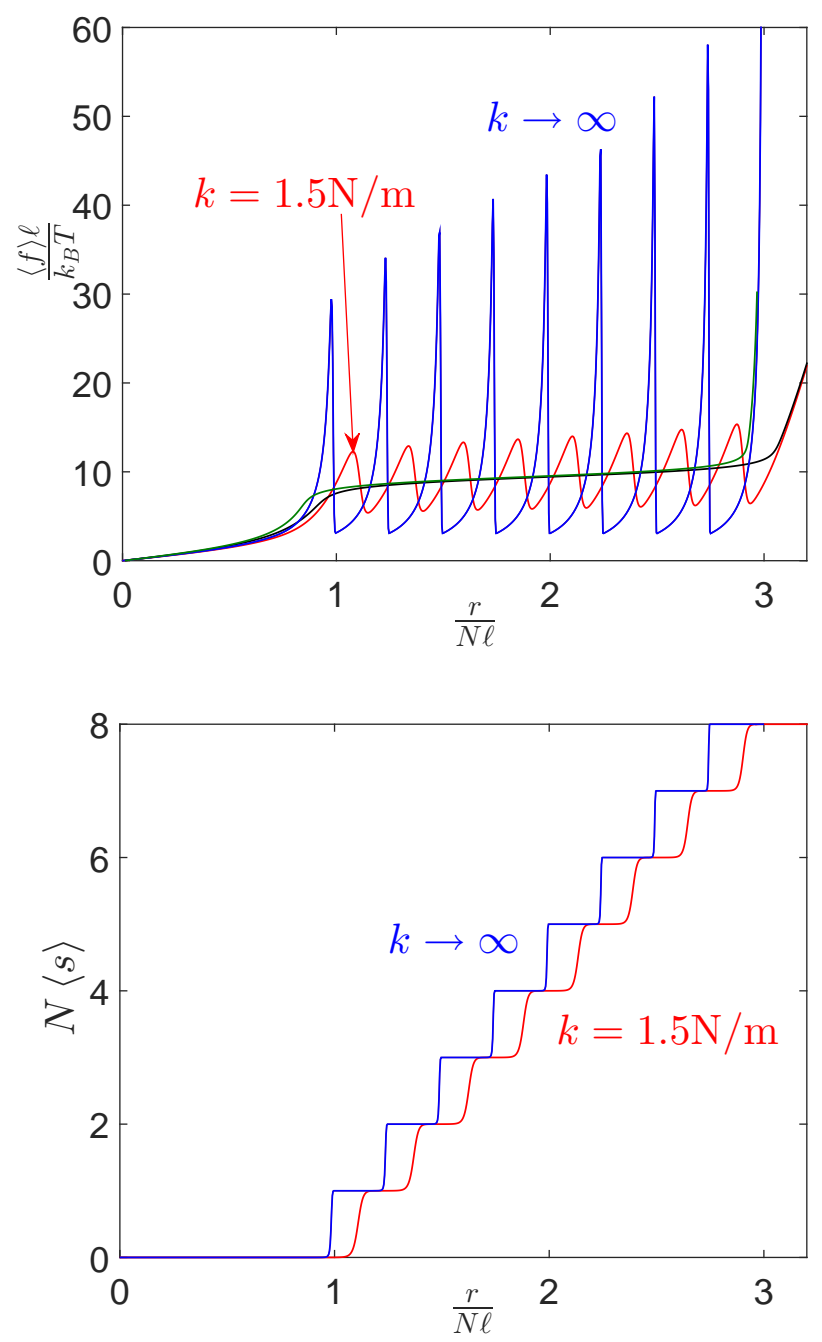

FIG. 5. Force-extension response (top panel) and average spin (bottom panel) for a system with extensibility $(k=1.5 \mathrm{~N} / \mathrm{m})$ and a system without extensibility $(k \rightarrow \infty)$. The calculation for the system with extensibility is based on Eq.(48) (red curves) while the system without extensibility has been studied through Eq.(49) (blue curves). In the top panel, we also added the Gibbs force-extension responses for both cases. We adopted the parameters $N=8, \ell=0.5 \times 10^{-9} \mathrm{~m}, T=300 \mathrm{~K}$, $\chi=3, \Delta E=20 k_{B} T=8.28 \times 10^{-20} \mathrm{~J}$ and $\mu=0$.

(see Section II). For instance, the force-extension response is given by the expression

$$
\langle f\rangle=\frac{\partial \mathcal{F}}{\partial r},
$$

and the average value of the spin variable is as follows

$$
N\langle s\rangle=\frac{\partial \mathcal{F}}{\partial \Delta E}=-\frac{\partial \mathcal{F}}{\partial \mu} .
$$

Of course, both $\langle f\rangle$ and $\langle s\rangle$ could be written in closed form by performing the indicated derivatives. However, for the sake of brevity, we omit this development and we show an example of application of Eqs.(50) and (51) in Fig.5, where we compare an elastic system described by Eq.(48) with a stiff one described by Eq.(49). In the top panel, we show the force extension curve for the stiff system with $k \rightarrow \infty$ and for the elastic one with $k=1.5 \mathrm{~N} / \mathrm{m}$. To better compare the responses, we also added the Gibbs force-extension responses for both cases. In the bottom panel, the average value of the spin variables is also represented for the stiff and the soft systems. These results prove a non-cooperative behavior characterized by a progressive unfolding of units in response to the increasing overall length. This behavior corresponds to a series of peaks in the force-extension curves and to a staircase function for the spin variable. Any peak or step corresponds to an unfolding process induced by the increasing extension. The overall behavior is therefore completely different from the Gibbs ensemble, where all units undergo the conformational transition at the same time. Anyway, for the soft system, we observe a sensibly reduced peak-to-peak force in the force extension curve and, correspondingly, a smoother transition in the steps of the average spin variable. Therefore, it is important to underline that relatively soft polymers (with small elastic constant) can have peak-to peak forces much smaller than ones predicted by the two-state freely jointed chain.

In Fig.6, one can find the force-extension response (top panel), the average spin variable (bottom-left panel) and the Helmholtz free energy (bottom-right panel) for a system with variable extensibility $k=0.4 j \mathrm{~N} / \mathrm{m}, \forall j=$ $1, \ldots, 6$. We can observe the progressive increase of the peak-to-peak force of each unfolding transition with increasing values of the elastic constant. At the same time, the transition are sharper for stiffer systems, as clearly visible in the average spin curves. The origin of the force peaks in the force extension curve can be highlighted in the Helmholtz free energy curves, which are characterized by a series of "smooth cusps" able to generate the force peaks by derivation with respect to the position.

A further analysis concerns the variation of the peakto-peak force with the elastic constant $k$ and the temperature $T$. In Fig.7, we can observe the shape of the first peaks for two temperatures $T=300 \mathrm{~K}$ and $T=750 \mathrm{~K}$ and for several values of $k=(0.3+0.15 n) \mathrm{N} / \mathrm{m}$, $n=0, \ldots, 18$. We can observe the strong reduction of the force peak with increasing elastic constant and/or temperature. Concerning the decrease of the peak with the temperature, we remark that the spinoidal behavior of the force extension curve (negative slope in some regions) is characterized by a critical temperature, defined as follows. For supercritical temperatures, the slope (or the effective stiffness) is always positive and for subcritical temperatures, it is negative in some regions. This concept of critical temperature for the chain within the Helmholtz ensemble is similar to the concept of Curie temperature governing the ferromagnetic-paramagnetic transition in magnetic materials. This point has been recently discussed in Refs.74-76. Interestingly enough, we notice that the chain with a negative effective stiffness 

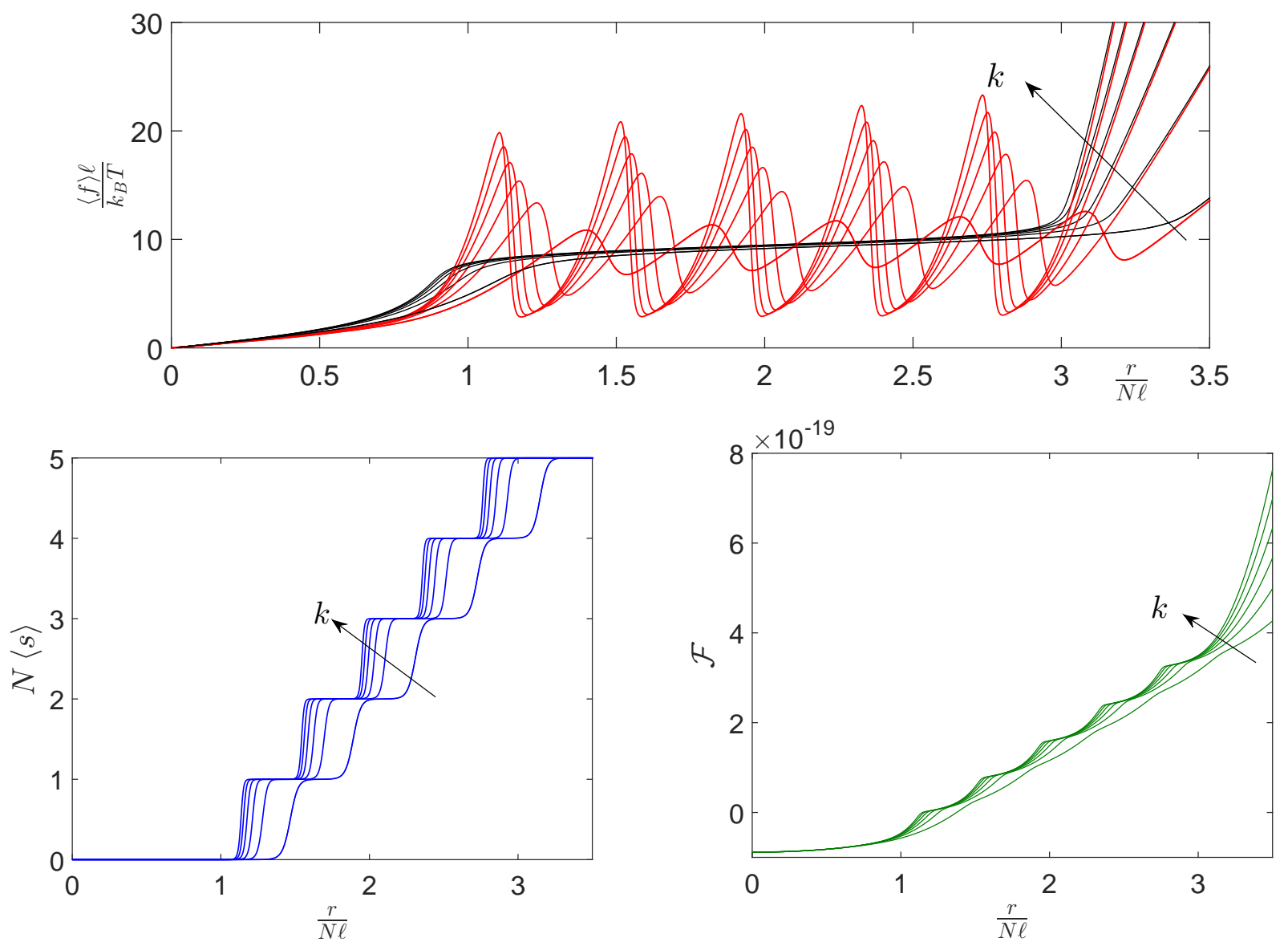

FIG. 6. Force-extension response (top panel), average spin variable (bottom-left panel) and Helmholtz free energy (bottomright panel) for a system with variable extensibility $k=0.4 j \mathrm{~N} / \mathrm{m}, \forall j=1, \ldots, 6$. The Helmholtz free energy $\mathcal{F}$ is represented in Joule. The calculation is based on Eq.(48). We also added the Gibbs force-extension responses (black curves) to facilitate the comparison. We adopted the parameters $N=5, \ell=0.5 \times 10^{-9} \mathrm{~m}, T=300 \mathrm{~K}, \chi=3, \Delta E=20 k_{B} T=8.28 \times 10^{-20} \mathrm{~J}$ and $\mu=0$.

(spinoidal behavior), exhibited in specific working conditions, can be viewed as a metamaterial ${ }^{74}$ and this property can be mimicked in bioinspired nanostructures. ${ }^{106}$ In Fig.8, one can find the the variation of the peak-to-peak force (as defined in Fig.7) in terms of elastic constant and temperature. We can see that the force peak becomes negligible for low values of $k$ and high values of $T$. This plot shows the importance of considering the correct value of $k$ for modeling the folding-unfolding processes of macromolecular chains. Indeed, the force peaks are typically considered as output of real experiments and their values are used to draw quantitative conclusions about mechanical and thermodynamic properties of the macromolecule.

To conclude, we show in Fig.9 the behavior of the system as a function of the number of units of the chain. This is an important analysis since concerns the validity of the ensembles equivalence in the thermodynamic limit. In Fig.9, one can find the results for $N$ from 2 to 8 for a system with an elastic constant $k=1 \mathrm{~N} / \mathrm{m}$. It is interesting to remark that for an increasing value of $N$, the peak-to-peak force is progressively reduced, confirm- ing the convergence of the Helmholtz ensemble to the Gibbs ensemble for $N \rightarrow \infty$. Indeed, if two ensembles are equivalent, the corresponding force-extension curves must coincide. This is perfectly coherent with known results concerning the ensembles equivalence in the thermodynamic limit, as discussed in recent literature. ${ }^{54,55}$

\section{CONCLUSIONS}

In this work, we discussed the spin variables approach used to describe the folding/unfolding behavior of chains with conformational transitions. We briefly reviewed the statistical mechanics of a chain within the Helmholtz (isometric) and Gibbs (isotensional) ensembles by proving that the additional spin variables do not modify the standard results of the macroscopic thermodynamics. In particular, the first and second principles hold on, provided that we slightly modify the average calculation method of microscopic quantities, namely by simply adding the sum over the spin variables $\vec{S}$ besides the classical integration over the variables $(\vec{q}, \vec{p})$. This approach is similar to 


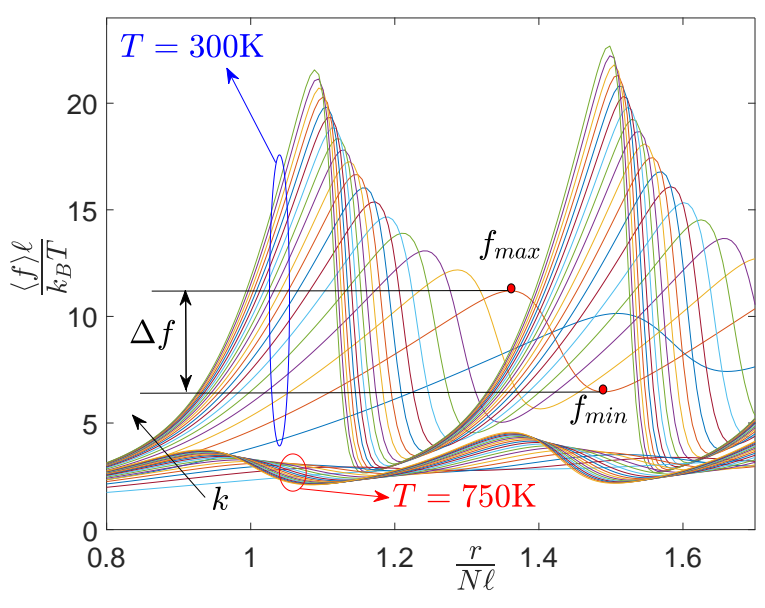

FIG. 7. Shape of the first peaks in the force extensione curves for two temperatures $T=300 \mathrm{~K}$ and $T=750 \mathrm{~K}$ and for several values of $k=(0.3+0.15 n) \mathrm{N} / \mathrm{m}, n=0, \ldots, 18$. One can see the exact definition of the peak-to-peak force for the first peak holding for each force-extension curve. We adopted the parameters $N=5, \ell=0.5 \times 10^{-9} \mathrm{~m}, \chi=3, \Delta E=8.28 \times 10^{-20} \mathrm{~J}$ and $\mu=0$.

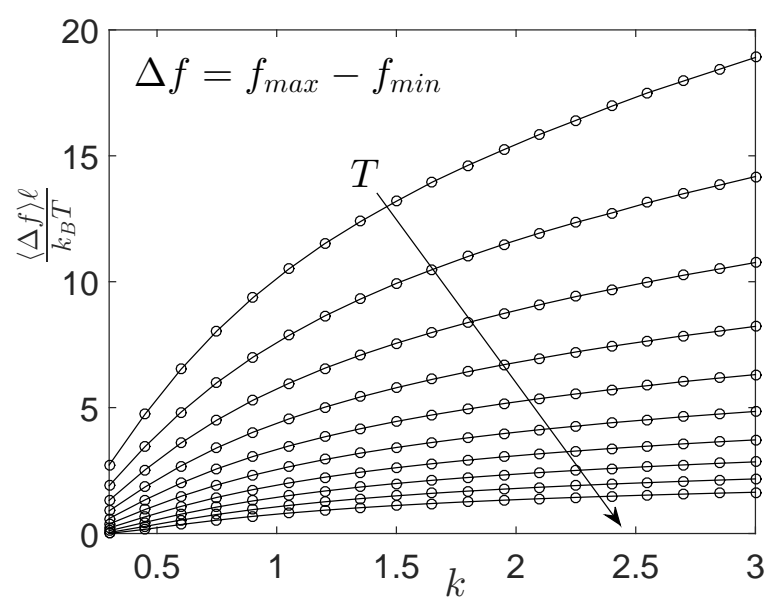

FIG. 8. Behavior of the peak-to-peak force defined in Fig.7 versus elastic constant and temperature. We varied the elastic constant in the range $k=(0.3+0.15 n) \mathrm{N} / \mathrm{m}, n=0, \ldots, 18$ and the temperature as $T=(300+50 m), m=0, \ldots, 9$. We observe the strong reduction of the force peak with the increasing of temperature and/or elastic constant. We adopted the parameters $N=5, \ell=0.5 \times 10^{-9} \mathrm{~m}, \chi=3, \Delta E=8.28 \times 10^{-20} \mathrm{~J}$ and $\mu=0$.

the introduction of the number of particles in the grand canonical ensemble of statistical mechanics as effective variable summed in the partition function and controlled by the chemical potential. The introduction of the spin variables approach allows therefore to describe the folding/unfolding of macromolecular chains by introducing simpler forms of the potential energy, thus facilitating the calculation of the partition functions. It means that each basin of the energy landscape can be approximated with a quadratic potential, which leads to an easier integration. The knowledge of the partition function is useful to directly determine the force-extension response of the chain within the statistical ensemble considered. Moreover, the average value of the spin variables as function of the externally controlled quantities (positions or forces) is useful to characterize and measure the cooperativeness (or synchronization) of the folding/unfolding processes. As an example, a potential energy with two energy wells separated by a given energy barrier can be substituted with a couple of quadratic potentials, which are, as discussed above, easier to handle from the mathematical point of view. The switching between the two quadratic wells is automatically controlled by the associated spin variable, as result of the externally applied boundary conditions. This procedure is based on an approximation since the above substitution of the original potential energy with two quadratic wells removes the portion of curve between the wells, corresponding to the energy barrier. If we work at thermodynamic equilibrium this assumption leads to accurate results (see also Ref.76) since the system explores only the bottom of the basins and does not feel the energy barrier between them. On the other hand, if we work within the out-of-equilibrium regime, the energy barrier plays a crucial role in determining the characteristic times of the process.

The idea of the spin variables has been exploited in the second part of the paper, where we analyzed in detail the case of a two-state freely jointed chain with extensible links. With respect to previous literature, we added in this work the extensibility of the units, which is an important point to describe real macromolecules but, at the same time, makes the mathematical problem considerably more involved.

Concerning the Gibbs ensemble, we obtained the exact partition function by direct integration, and then we introduced an approximation yielding a simpler expression useful for further developments. We thoroughly verified the validity of this approximation in the standard ranges of variability of the parameters. The partition function is then used to calculate the elastic response of the system and the average value of the spin variable. This quantity exhibits a transition, for a given applied force, describing the cooperative (or synchronized) behavior of the Gibbs response.

The following development concerns the Helmholtz ensemble. In this case, the direct calculation of the partition function can not be done because of the isometric condition, which generates an effective interaction among the units of the chain. It means that the partition function can not be factorized as in the case of the Gibbs ensemble, where the units are de facto independent. So, the adopted technique is based on the property stating that the Gibbs and Helmholtz partition functions are connected through a Laplace transform. This property is exploited in classical literature to prove the equivalence of 

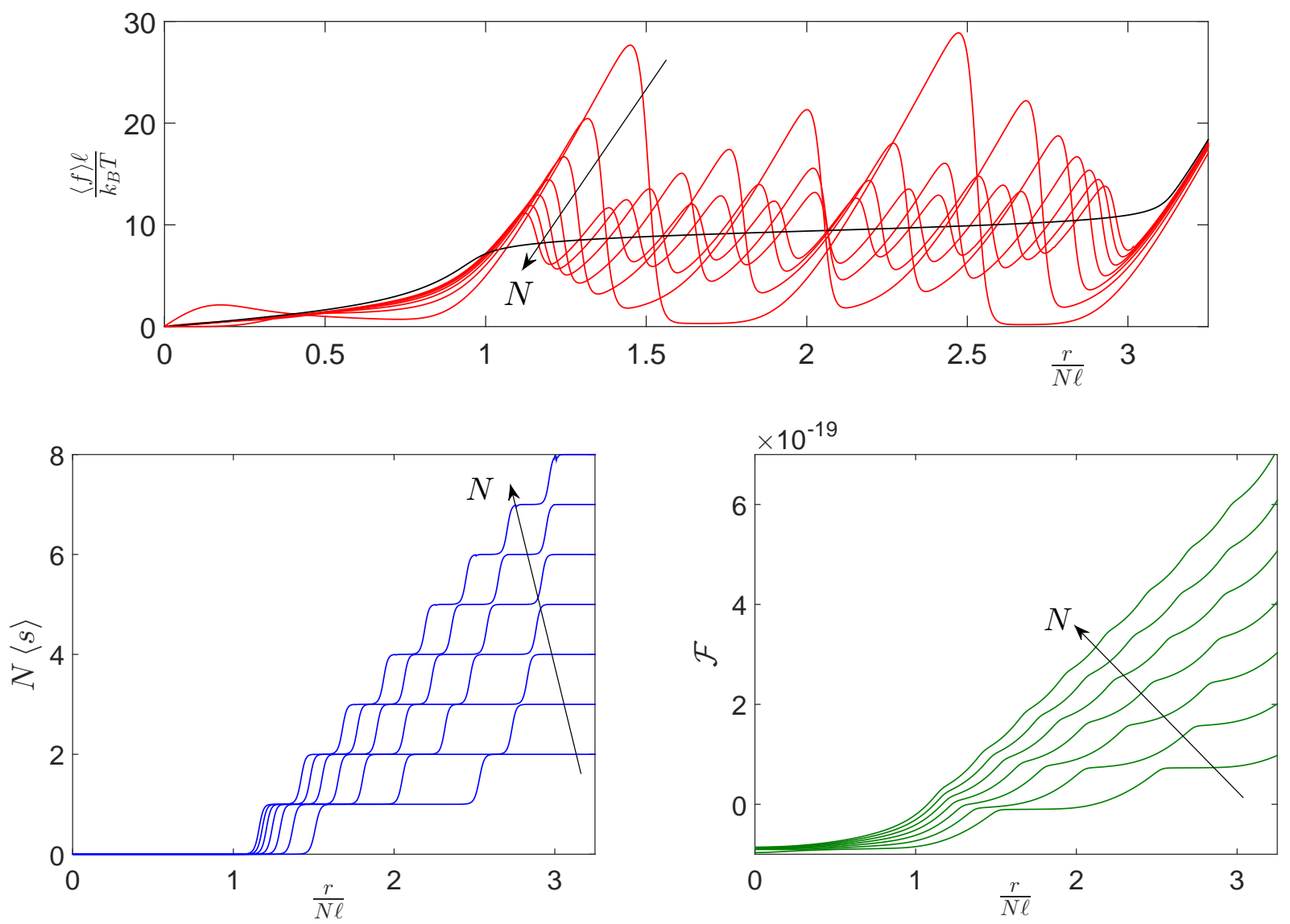

FIG. 9. Force-extension response (top panel), average spin variable (bottom-left panel) and Helmholtz free energy (bottomright panel) for a system with a variable number of units $N=2,3,4,5,6,7$, and 8. The Helmholtz free energy $\mathcal{F}$ is represented in Joule. The calculation is based on Eq.(48). We also added the Gibbs force-extension responses (black curves) to facilitate the comparison. We adopted the parameters $k=1 \mathrm{~N} / \mathrm{m}, \ell=0.5 \times 10^{-9} \mathrm{~m}, T=300 \mathrm{~K}, \chi=3, \Delta E=20 k_{B} T=8.28 \times 10^{-20} \mathrm{~J}$ and $\mu=0$.

the ensembles in the thermodynamic limit. ${ }^{33,37}$ Indeed, the Laplace transform between $Z_{G}$ and $Z_{H}$ allows one to prove that the Legendre transform holds between the free energies $\mathcal{G}$ and $\mathcal{F}$ for large values of $N$. Alternatively, we use here the Laplace property between the partition functions to find an explicit form of $Z_{H}$ for a system with an arbitrary elastic constant and an arbitrary number of units in the chain. Interestingly enough, we obtain the closed form expression for this partition function in terms of the Hermite elements with negative index. They represent the natural generalization of the classical Hermite polynomials $H_{n}(z)$, obtained by considering negative values for $n$. Although they have been introduced in the mathematical literature, ${ }^{107}$ to the author knowledge this is the first application to a notable physical problem. The knowledge of the partition function allows the determination of the force-extension curve and of the average value of the spin variable. The latter exhibits a stepwise behavior, well explaining the non-cooperative (or non-synchronized) nature of the Helmholtz response. In conclusion, the conducted analysis has generalized the results on the two-state freely jointed chain $^{76}$ to the case with a finite extensibility of the macromolecule domains.

It is important to discuss in more detail the experimental implications of the analysis conducted in our work. We underline that both the experiments conducted with soft devices (Gibbs ensemble) and hard devices (Helmholtz ensemble) can be relevant to obtain quantitative properties of individual macromolecules. For instance, concerning the soft devices, the following information can be extracted from one experiment:

- from an experimental force-extension curve similar to that shown in Fig.3 (left panel), we can easily measure the transition force $f^{*}$ (height of the plateau $)$ and the transition extension $(\chi-1) \ell$ (width of the plateau), which are structural properties of the molecule with conformational transitions;

- most importantly, from previous quantities, we can easily determine the energy jump $\Delta E$ between the folded (native) and unfolded states of the units of 
the chain, a crucial property of the system, which is difficult to measure without force-spectroscopy techniques; this evaluation is based on the relation $f^{*}=\frac{\Delta E}{(\chi-1) \ell}$, discussed in Section III.

Concerning the hard devices, the following issues clarify the relation with experiments:

- first of all, we can easily measure the number of units of the chain, which directly corresponds to the number of peaks in the force-extension curve;

- in order to explore the whole range of the extension of the chain, the typical experimental protocol uses a fixed pulling velocity of the moving extremity of the molecule; correspondingly, the peaks of force depend on the pulling velocity, and this dependence can be used to evaluate the height of the energy barrier $\mathcal{A}$ between the folded (native) and unfolded states; further details can be found in recent literature; ${ }^{108,109}$

- the Helmholtz response is crucial to investigate the cooperativity. To begin, we clarify the term cooperativity, which is used with two different meanings in this context. Firstly, when we describe the different behavior of the Gibbs and Helmholtz ensembles, we observe a cooperative versus non-cooperative response, but it should be better to use the term synchronization since these distinct responses are generated by the different boundary conditions (imposed force or imposed extension) and not by actual unit-unit interactions. Secondly, the real cooperativity is observed when the transition of one element affects the transition of the others (this process is induced by interactions among units). ${ }^{83}$ So said, the Helmholtz response of a repeat chain (for instance repeat protein) is very useful to investigate the real cooperativity generated by the interactions. Indeed, both the long- and short-range interactions among domains of a protein strongly modify the force peaks observed in the Helmholtz force-extension curve. The sequence of transitions defines the unfolding pathway and the force peak of each unit is small if there is a strong interaction with previously unfolded units and is large if the interaction is negligible. Therefore, the cooperativity is observed through a heterogeneous hierarchy of force peaks. As discussed in Section II, this phenomenon has been observed in spectrin ${ }^{84}$ and filamin A. ${ }^{85}$ The understanding of cooperativity can be improved by implementing the Ising model within the potential energy of the system (see below) and by comparing the results with the behavior of other repeat protein structures. ${ }^{86-88}$

- an important factor, which affects the sawtoothlike force-extension response under isometric conditions is the intrinsic elasticity of the units; here, we clearly proved that a weak stiffness of the chain may strongly reduce the force peaks of the response (see, e.g., Fig.6), thus reducing the possibility of using hard devices to study cooperativity and other conformational properties. It means that the best results can be obtained with quite stiff macromolecules.

The spin variable approach will be adopted in the near future to study more complicated situations. As anticipated in Section II, important energy terms that must be taken into consideration concern the interaction among the folding/unfolding states of the units (e.g. through an Ising-like interaction term), and the semi-flexibility of real chains (e.g. through the classical WLC scheme). It is interesting to note that an interaction term based on a Ising mechanism can be only considered after the introduction of the spin variables for the units of the chain. This point makes the spin variables approach suitable and appropriate for such a generalization. Also, the consideration of the semi-flexibility through the WLC model is important to correctly describe the properties of dsDNA and several proteins, which show a given persistence length. Another perspective concerns the dynamics of the folding/unfolding process, which must be studied in the context of the out-of-equilibrium statistical mechanics. Recently, the dynamics of systems with a multibasin energy landscape has been studied. ${ }^{64,79,108,109}$ This analysis should be completed by investigating the dynamics of the whole system, described by continuous (mechanical) coordinates and discrete spin variables. It is worth noting that the spin variable approach can be used in this context for decoupling the mechanical characteristic times (induced by the spring-like behavior of wells) from the times induced by the transition rates between the wells, which depend on the energy barrier as classically described by the Kramers theory. ${ }^{78}$

Finally, it is interesting to remark that the statistical mechanics and the dynamics of systems with multi-well energy are useful to model other physical situations including, but not limited to, cell adhesion, macromolecular hairpins, skeletal muscles, ferromagnetic alloys, nanoindented substrates and plastic materials. ${ }^{71,75,77,80}$

\section{ACKNOWLEDGMENTS}

The authors thank Tom Lindstrøm for providing the original copy of Ref.107, concerning the Hermite elements with negative index. We also acknowledge the region "Hauts de France" for the financial support under project MEPOFIB. 


\section{Appendix A: An integral calculation}

To perform the calculation of the Helmholtz partition function we have to determine the value of

$$
I_{m}(a, b)=\int_{\Gamma} e^{i a y} e^{-b y^{2}} \frac{d y}{y^{m}},
$$

where $a \in \mathbb{R}, b>0, m \in \mathbb{N}$ and the path $\Gamma$ is given in Fig.4. To this aim, we begin to introduce the closed paths ABCD shown in Fig.10. To understand the suitability of this approach, we firstly consider the segment CD on the paths shown in Fig.10. Here, we have $y=s+i \beta$ where $s \in \mathbb{R}$ is a variable and $\beta \in \mathbb{R}$ is a constant that we will determine with the purpose of simplifying the integral calculation. Therefore, we get

$$
\begin{aligned}
& \int_{C D} e^{i a y} e^{-b y^{2}} \frac{d y}{y^{m}} \\
= & -\int_{-R}^{+R} e^{i a(s+i \beta)} e^{-b(s+i \beta)^{2}} \frac{d s}{(s+i \beta)^{m}} \\
= & -e^{-a \beta} e^{b \beta^{2}} \int_{-R}^{+R} e^{i s(a-2 b \beta)} e^{-b s^{2}} \frac{d s}{(s+i \beta)^{m}},
\end{aligned}
$$

and we can let $\beta=a /(2 b)$ to remove the imaginary exponential from the integral. Hence, the result can be written as

$$
\int_{C D} e^{i a y} e^{-b y^{2}} \frac{d y}{y^{m}}=-e^{-\frac{a^{2}}{4 b}} \int_{-R}^{+R} \frac{e^{-b s^{2} d s}}{\left(s+i \frac{a}{2 b}\right)^{m}}
$$

and, applying the limit for $R \rightarrow \infty$ and the change of variable $\eta=\sqrt{b} s$, it assumes the final form

$\lim _{R \rightarrow \infty} \int_{C D} e^{i a y} e^{-b y^{2}} \frac{d y}{y^{m}}=-b^{\frac{m-1}{2}} e^{-\frac{a^{2}}{4 b}} \int_{-\infty}^{+\infty} \frac{e^{-\eta^{2} d \eta}}{\left(\eta+i \frac{a}{2 \sqrt{b}}\right)^{m}}$.

This expression, of course, is valid for both negative and positive values of $a$.

For the calculation of the integral in Eq.(A1), we have to consider two separate cases. If $a>0$ or $\beta>0$, the path shown in Fig.10(a) does not contain the origin of the complex plane and therefore the function to integrate is holomorphic within the path. In this situation, we can directly apply the Cauchy theorem stating that

$$
\left(\int_{A B}+\int_{B C}+\int_{C D}+\int_{D A}\right) e^{i a y} e^{-b y^{2}} \frac{d y}{y^{m}}=0 .
$$

Now, in the limit for $R \rightarrow \infty$, it is not difficult to prove that the integrals on the segments BC and DA vanish and we get the first result for $a>0$

$$
I_{m}(a, b)=b^{\frac{m-1}{2}} e^{-\frac{a^{2}}{4 b}} \int_{-\infty}^{+\infty} \frac{e^{-\eta^{2} d \eta}}{\left(\eta+i \frac{a}{2 \sqrt{b}}\right)^{m}} .
$$

If $a<0$ or $\beta<0$, the path shown in Fig. 10(b) contains the origin of the complex plane, which corresponds to a
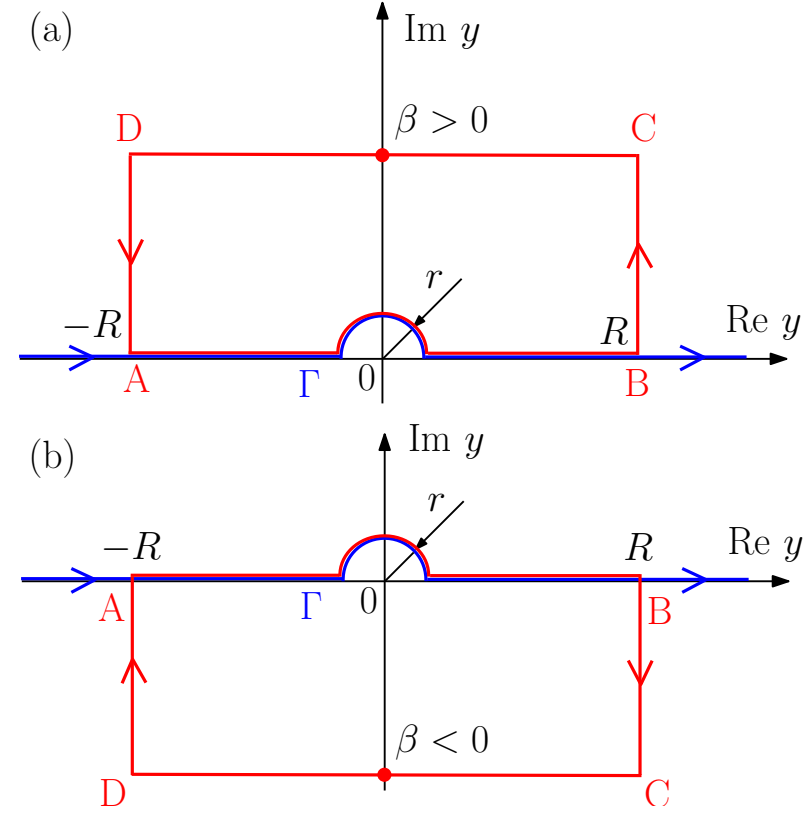

FIG. 10. Definition of the contours ABCD on the complex plane for $\beta>0$ (a) and $\beta<0$ (b).

singular point of the function to integrate. Hence, the residue theorem delivers

$$
\begin{aligned}
& \left(\int_{A B}+\int_{B C}+\int_{C D}+\int_{D A}\right) e^{i a y} e^{-b y^{2}} \frac{d y}{y^{m}} \\
& =-2 \pi i \operatorname{Res}\left\{\frac{e^{i a y} e^{-b y^{2}}}{y^{m}}, 0\right\},
\end{aligned}
$$

where the residue can be easily calculated since the singular point is a pole or order $m$

$$
\operatorname{Res}\left\{\frac{e^{i a y} e^{-b y^{2}}}{y^{m}}, 0\right\}=\frac{1}{(m-1) !} \frac{d^{m-1}}{d y^{m-1}}\left(e^{i a y} e^{-b y^{2}}\right)_{y=0} .
$$

The derivative in Eq.(A8) can be elaborated through the general rule

$$
\frac{d^{m-1}}{d y^{m-1}}[f(y) g(y)]=\sum_{k=0}^{m-1}\left(\begin{array}{c}
m-1 \\
k
\end{array}\right) \frac{d^{k} f(y)}{d y^{k}} \frac{d^{m-1-k} g(y)}{d y^{m-1-k}} .
$$

If $g(y)=e^{i a y}$, we simply have

$$
\frac{d^{m-1-k} g(y)}{d y^{m-1-k}}=(i a)^{m-1-k} e^{i a y} .
$$

On the other hand, if $f(y)=e^{-b y^{2}}$, the calculation of the derivative of order $k$ is more involved. One method is based on the well-known integral

$$
\int_{-\infty}^{+\infty} e^{-\frac{x^{2}}{4 b}} e^{i x y} d x=\sqrt{4 \pi b} e^{-b y^{2}}
$$


which can be exploited as follows

$$
\frac{d^{k} f(y)}{d y^{k}}=\frac{1}{\sqrt{4 \pi b}} \int_{-\infty}^{+\infty} e^{-\frac{x^{2}}{4 b}}(i x)^{k} e^{i x y} d x .
$$

Hence, if we consider $y=0$, we get

$$
\left(\frac{d^{k} f(y)}{d y^{k}}\right)_{y=0}=\frac{i^{k}}{\sqrt{4 \pi b}} \int_{-\infty}^{+\infty} x^{k} e^{-\frac{x^{2}}{4 b}} d x .
$$

Then, we observe that the result is different from zero only for $k$ even. In particular, we eventually obtain

$$
\left(\frac{d^{k} f(y)}{d y^{k}}\right)_{y=0}= \begin{cases}\frac{(-4 b)^{n}}{\sqrt{\pi}} \Gamma\left(n+\frac{1}{2}\right) & \text { if } k=2 n \\ 0 & \text { if } k=2 n+1,\end{cases}
$$

where $\Gamma(z)$ is the Euler Gamma function. By combining Eqs.(A9), (A10) and (A14), we can rewrite the residue defined in Eq.(A8) as

$$
\begin{aligned}
& \operatorname{Res}\left\{\frac{e^{i a y} e^{-b y^{2}}}{y^{m}}, 0\right\} \\
& =\sum_{k=0}^{m-1} \frac{(i a)^{m-1-k}}{k !(m-1-k) !} \frac{(-4 b)^{k / 2}}{2 \sqrt{\pi}} \Gamma\left(\frac{k+1}{2}\right)\left[1+(-1)^{k}\right] .
\end{aligned}
$$

Therefore, the residue theorem written in the form of Eq.(A7), and considered for $R \rightarrow \infty$, gives the final result for $a<0$

$$
\begin{aligned}
& I_{m}(a, b)=b^{\frac{m-1}{2}} e^{-\frac{a^{2}}{4 b}} \int_{-\infty}^{+\infty} \frac{e^{-\eta^{2} d \eta}}{\left(\eta+i \frac{a}{2 \sqrt{b}}\right)^{m}} \\
& -\sum_{k=0}^{m-1} \frac{2 \pi i(i a)^{m-1-k}}{k !(m-1-k) !} \frac{(-4 b)^{k / 2}}{2 \sqrt{\pi}} \Gamma\left(\frac{k+1}{2}\right)\left[1+(-1)^{k}\right] .
\end{aligned}
$$

To conclude, Eq.(A6) for $a>0$ and Eq.(A16) for $a<0$ represent the integral $I_{m}(a, b)$ defined in Eq.(A1) in terms of a second integral, which is the subject of the following Appendix B.

\section{Appendix B: The Hermite elements with negative index}

First of all, we observe that the integral

$$
J_{m}(x)=\int_{-\infty}^{+\infty} \frac{e^{-\eta^{2}} d \eta}{(\eta+i x)^{m}}
$$

appearing in Eqs.(A6) and (A16) with $x=a /(2 \sqrt{b})$, is simpler than $I_{m}(a, b)$ since it is calculated over the real axis and there is no imaginary exponent in the function to integrate. To approach its calculation, we recall the following integral representation of the Hermite polynomials ${ }^{93,94}$

$$
\begin{aligned}
H_{m}(z) & =\frac{2^{m}}{\sqrt{\pi}} \int_{-\infty}^{+\infty}(i \eta+z)^{m} e^{-\eta^{2}} d \eta \\
& =\frac{(2 i)^{m}}{\sqrt{\pi}} \int_{-\infty}^{+\infty}(\eta-i z)^{m} e^{-\eta^{2}} d \eta .
\end{aligned}
$$

If we compare $J_{m}$ with the last representation of $H_{m}$, we note that $J_{m}$ is formally related to $H_{-m}$, which represents an Hermite element with negative index. We underline that the representation of the Hermite polynomials in Eq.(B2) makes sense also for negative values of $m$. However, we will see that these functions are not polynomials but they exhibit several properties similar to those of the classical Hermite polynomials. To the author knowledge, these functions have been firstly introduced and studied in Ref.107 but no other analysis or application can be found in the literature. Anyway, by replacing $m$ with $-m$ in Eq.(B2), we define

$$
H_{-m}(z)=\frac{1}{(2 i)^{m} \sqrt{\pi}} \int_{-\infty}^{+\infty} \frac{e^{-\eta^{2}} d \eta}{(\eta-i z)^{m}}
$$

and we introduce their properties. Following Ref.107, we firstly analyse the first element $H_{-1}(z)$. We simply have

$$
\begin{aligned}
H_{-1}(z) & =\frac{1}{2 i \sqrt{\pi}} \int_{-\infty}^{+\infty} \frac{e^{-\eta^{2}} d \eta}{\eta-i z} \\
& =\frac{1}{2 i \sqrt{\pi}} \int_{-\infty}^{+\infty} \frac{\eta+i z}{\eta^{2}+z^{2}} e^{-\eta^{2}} d \eta \\
& =\frac{1}{2 \sqrt{\pi}} \int_{-\infty}^{+\infty} \frac{z}{\eta^{2}+z^{2}} e^{-\eta^{2}} d \eta .
\end{aligned}
$$

Indeed, the imaginary part is zero since it corresponds to an odd function integrated over the symmetric interval $(-\infty,+\infty)$. From Eq.(B4), we deduce that $H_{-1}(z)$ is an odd function of $z$. Then we can study $H_{-1}(z)$ for $z>0$. Under this hypothesis, we define $u=\eta / z$ and we obtain

$$
H_{-1}(z)=\frac{1}{2 \sqrt{\pi}} \int_{-\infty}^{+\infty} \frac{e^{-u^{2} z^{2}} d u}{1+u^{2}} \quad(z>0),
$$

from which we easily deduce the particular value $\lim _{z \rightarrow 0^{+}} H_{-1}(z)=\sqrt{\pi} / 2$. In addition, Eq.(B5) allows us to get a differential equation for $H_{-1}(z)(z>0)$. By differentiation, we have

$$
\begin{aligned}
\frac{d}{d z} H_{-1}(z) & =-\frac{z}{\sqrt{\pi}} \int_{-\infty}^{+\infty} \frac{u^{2} e^{-u^{2} z^{2}} d u}{1+u^{2}} \\
& =-\frac{z}{\sqrt{\pi}} \int_{-\infty}^{+\infty}\left(\frac{1+u^{2}}{1+u^{2}}-\frac{1}{1+u^{2}}\right) e^{-u^{2} z^{2}} d u \\
& =-1+2 z H_{-1}(z) .
\end{aligned}
$$

Now, the differential equation $H_{-1}^{\prime}(z)=-1+2 z H_{-1}(z)$ is linear and can be directly solved for $z>0$ with the initial condition $H_{-1}\left(0^{+}\right)=\sqrt{\pi} / 2$ proved above. A straightforward calculation yields

$$
H_{-1}(z)=\frac{\sqrt{\pi}}{2} e^{z^{2}}[1-\operatorname{erf}(z)], \quad z>0 .
$$

Since $H_{-1}(z)$ is odd, i.e. $H_{-1}(-z)=-H_{-1}(z)$, we can also calculate its values for $z<0$. By means of the first two functions $H_{0}(z)=1 \forall z$ and $H_{-1}(z)$ given in Eq.(B7), 
we can determine all other Hermite elements with negative index through the following recursive formula

$$
H_{-(n+1)}(z)=\frac{1}{2 n} H_{-(n-1)}(z)-\frac{z}{n} H_{-n}(z) .
$$

Following again Ref.107, Eq.(B8) can be proved as follows

$$
\begin{aligned}
H_{-(n-1)}(z)= & \frac{1}{(2 i)^{n-1} \sqrt{\pi}} \int_{-\infty}^{+\infty} \frac{e^{-\eta^{2}} d \eta}{(\eta-i z)^{n-1}} \\
= & \frac{1}{(2 i)^{n-1} \sqrt{\pi}} \int_{-\infty}^{+\infty} \frac{\eta-i z}{(\eta-i z)^{n}} e^{-\eta^{2}} d \eta \\
= & \frac{1}{(2 i)^{n-1} \sqrt{\pi}} \int_{-\infty}^{+\infty} \frac{\eta e^{-\eta^{2}} d \eta}{(\eta-i z)^{n}} \\
& -\frac{i z}{(2 i)^{n-1} \sqrt{\pi}} \int_{-\infty}^{+\infty} \frac{e^{-\eta^{2}} d \eta}{(\eta-i z)^{n}} .
\end{aligned}
$$

Now, the first integral in the result of Eq.(B9) can be elaborated by parts, eventually obtaining

$$
\int_{-\infty}^{+\infty} \frac{\eta e^{-\eta^{2}} d \eta}{(\eta-i z)^{n}}=-\frac{n}{2} \int_{-\infty}^{+\infty} \frac{e^{-\eta^{2}} d \eta}{(\eta-i z)^{n+1}}
$$

Therefore, Eq.(B9) becomes $H_{-(n-1)}(z)=$ $2 n H_{-(n+1)}(z)+2 z n H_{-n}(z)$, which is equivalent to Eq.(B8). This recursive law allows us to affirm that $H_{-n}(z)$ is odd if $n$ is odd and that $H_{-n}(z)$ is even if $n$ is even. We underline that the Hermite elements with $n<0$ are not polynomials, contrarily to the classical Hermite polynomials, defined with $n>0$. Many other interesting properties of this sequence of functions can be found in Ref.107. For the sake of completeness, the first functions are reported below for $z>0$

$$
\begin{aligned}
& H_{0}(z)=1 \\
& H_{-1}(z)=\frac{\sqrt{\pi}}{2} e^{z^{2}} \operatorname{erfc}(z) \\
& H_{-2}(z)=\frac{1}{2}-\frac{\sqrt{\pi}}{2} z e^{z^{2}} \operatorname{erfc}(z), \\
& H_{-3}(z)=-\frac{1}{4} z+\frac{\sqrt{\pi}}{4}\left(\frac{1}{2}+z^{2}\right) e^{z^{2}} \operatorname{erfc}(z), \\
& H_{-4}(z)=\frac{1}{12}\left(1+z^{2}\right)-\frac{\sqrt{\pi}}{4} z\left(\frac{1}{2}+\frac{1}{3} z^{2}\right) e^{z^{2}} \operatorname{erfc}(z)
\end{aligned}
$$

where $\operatorname{erfc}(z)=1-\operatorname{erf}(z)$ is the complementary error function. Their plot can be found in Fig.11. From the computational point of view, the calculation of $e^{z^{2}} \operatorname{erfc}(z)$ must be done with the following asymptotic expansion (for $z>4)^{93,94}$

$$
\sqrt{\pi} z e^{z^{2}} \operatorname{erfc}(z) \sim 1+\sum_{m=1}^{M}(-1)^{m} \frac{1 \cdot 3 \ldots(2 m-1)}{\left(2 z^{2}\right)^{m}},(
$$

with a sufficiently large value of $M$.

Coming back to the calculation of the integral defined in Eq.(B1), we have finally obtained the following result, written in terms of the functions $H_{-m}(z)$

$$
J_{m}(x)=(2 i)^{m} \sqrt{\pi} H_{-m}(-x) .
$$

The integral $I_{m}(a, b)$ defined in Eq.(A1) can be therefore solved by Eq.(A6) for $a>0$ and by Eq.(A16) for $a<0$,

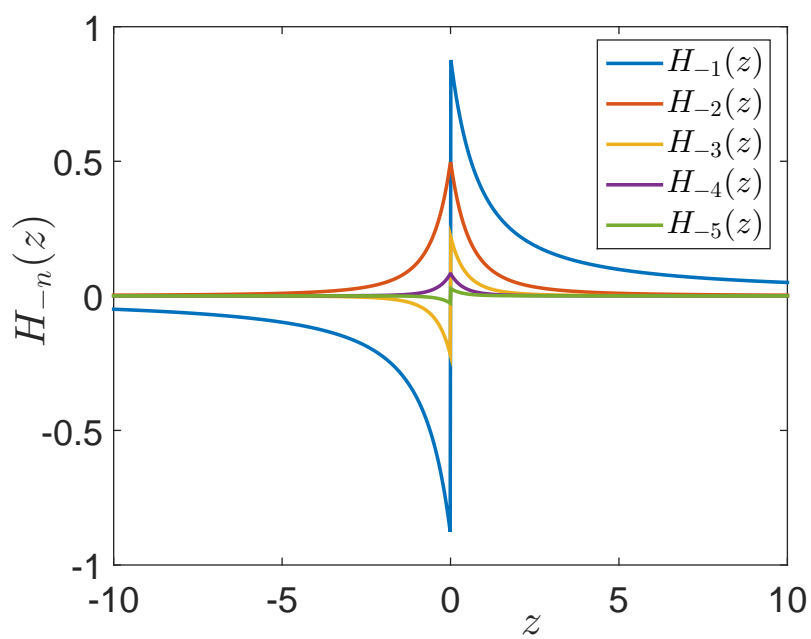

FIG. 11. Plot of the first Hermite elements with negative index. We observe that $H_{-n}(z)$ is odd if $n$ is odd and that that $H_{-n}(z)$ is even if $n$ is even.

rewritten and unified here in the compact form

$I_{m}(a, b)=\sqrt{\pi}\left\{b^{\frac{m-1}{2}} e^{-\frac{a^{2}}{4 b}}(2 i)^{m} H_{-m}\left(-\frac{a}{2 \sqrt{b}}\right)\right.$

$\left.+\mathbf{1}(-a) \sum_{k=0}^{m-1} \frac{(i a)^{m-1-k}(-4 b)^{k / 2}}{i k !(m-1-k) !} \Gamma\left(\frac{k+1}{2}\right)\left[1+(-1)^{k}\right]\right\}$,

where $\mathbf{1}(x)$ represents the Heaviside step function, defined as $\mathbf{1}(x)=1$ if $x \geq 0$, and $\mathbf{1}(x)=0$ if $x<0$.

\section{REFERENCES}

${ }^{1}$ W. A. Linke, H. Granzier and M. S. Z. Kellermayer (Eds.), Mechanics of Elastic Biomolecules (Springer Science, Dordrecht, 2003).

${ }^{2}$ A. Noy (Ed.), Handbook of Molecular Force Spectroscopy (Springer Science, New York, 2008).

${ }^{3}$ A. R. Bizzarri and S. Cannistraro (Eds.), Dynamic Force Spectroscopy and Biomolecular Recognition (CRC Press, Boca Raton, 2012).

${ }^{4}$ C. Bustamante, J. Liphardt and F. Ritort, Physics Today 58, 43 (2005).

${ }^{5}$ E. Dieterich, J. Camunas-Soler, M. Ribezzi-Crivellari, U. Seifert, and F. Ritort, Phys. Rev. E 94, 012107 (2016).

${ }^{6}$ T. R. Strick, M.-N. Dessinges, G. Charvin, N. H. Dekker, J.-F. Allemand, D. Bensimon and V. Croquette, Rep. Progr. Phys. 66, 1 (2003).

${ }^{7}$ F. Ritort, J. Phys.: Condens. Matter 18, R531 (2006).

${ }^{8}$ F. Cleri, Sci. Model. Simul. 15, 369 (2008).

${ }^{9}$ K. C. Neuman, A. Nagy, Nature Meth. 5, 491 (2008).

${ }^{10}$ S. Kumar, M. S. Li, Physics Reports 486, 1 (2010).

${ }^{11}$ H. Miller, Z. Zhou, J. Shepherd, A. J. M. Wollman, and M. C. Leake, Rep. Prog. Phys. 81, 024601 (2018). 
${ }^{12}$ T. E. Fisher, A. F. Oberhauser, M. Carrion-Vazquez, P. E. Marszalek, and J. M. Fernandez, Trends Biochem. Sci. 24, 379 (1999).

${ }^{13}$ H. Li, A. F. Oberhauser, S. B. Fowler, J. Clarke, and J. M. Fernandez, Proc. Nat. Acad. Sci. 97, 6527 (2000).

${ }^{14}$ A. Imparato, F. Sbrana, and M. Vassalli, Europhys Lett. 82, 58006 (2008).

${ }^{15}$ T. Hoffmann and L. Dougan, Chem. Soc. Rev. 41, 4781 (2012).

${ }^{16}$ K. M. Tych, T. Hoffmann, D. J. Brockwell, and L. Dougan, Soft Matter 9, 9016 (2013).

${ }^{17}$ M. Bonin, R. Zhu, Y. Klaue, J. Oberstrass, E. Oesterschulze, and W. Nellen, Nucleic Acids Res. 30, e81 (2002).

${ }^{18}$ J. Lipfert, G. M. Skinner, J. M. Keegstra, T. Hensgens, T. Jager, D. Dulin, M. Kber, Z. Yu, S. P. Donkers, F.-C. Chou, R. Das, and N. H. Dekker, Proc. Nat. Acad. Sci. 111, 15408 (2014).

${ }^{19}$ S. B. Smith, L. Finzi, and C. Bustamante, Science 258, 1122 (1992).

${ }^{20}$ J. F. Marko, E. D. Siggia, Macromolecules 28, 8759 (1995).

${ }^{21}$ S. M. Smith, Y. Cui, C. Bustamante, Science 271, 795 (1996).

${ }^{22}$ C. Bustamante, Z. Bryant, and S. B. Smith, Nature 421, 423 (2003).

${ }^{23}$ K. R. Chaurasiya, T. Paramanathan, M. J. McCauley, and M. C. Williams, Phys. Life Rev. 7, 299 (2010).

${ }^{24}$ S. Bezrukavnikov, A. Mashaghi, R. J. van Wijk, C. Gu, L. J. Yang, Y. Q. Gao, and S. J. Tans, Soft Matter 10, 7269 (2014).

${ }^{25}$ C. Yamahata, D. Collard, B. Legrand, T. Takekawa, M. Kumemura, G. Hashiguchi, H. Fujita, J. Microelectromech. Syst. 17, 623 (2008).

${ }^{26}$ F. Manca, S. Giordano, P. L. Palla, and F. Cleri, Phys. Rev. Lett. 113, 255501 (2014).

${ }^{27}$ G. Perret, T. Lacornerie, F. Manca, S. Giordano, M. Kumemura, N. Lafitte, L. Jalabert, M. C. Tarhan, E. F. Lartigau, F. Cleri, H. Fujita, and D. Collard, Microsystems \& Nanoengineering 2, 16062 (2016).

${ }^{28}$ W. Bialek, Rep. Prog. Phys. 81, 012601 (2018).

${ }^{29}$ M. V. Volkenstein, Configurational Statistics of Polymer Chains (Interscience, New York, 1963).

${ }^{30} \mathrm{H}$. Kleinert, Path Integrals in Quantum Mechanics, Statistics and Polymer Physics (World Scientific, London, 1990).

${ }^{31}$ R. H. Boyd, P. J. Phillips, The Science of Polymer Molecules (Cambridge University Press, Cambridge, 1993).

${ }^{32}$ M. Doi, Introduction to Polymer Physics (Clarendon, Oxford, 1996).

${ }^{33}$ J.H. Weiner, Statistical Mechanics of Elasticity (Dover Publication Inc., New York, 2002).

${ }^{34}$ M. Rubinstein, R. H. Colby, Polymer Physics (Oxford University Press, New York, 2003).

${ }^{35}$ R.G. Winkler, J. Chem. Phys. 118, 2919 (2003).

${ }^{36}$ G. Glatting, R.G. Winkler, P. Reineker, Macromolecules 26, 6085 (1993).
${ }^{37}$ F. Manca, S. Giordano, P. L. Palla, R. Zucca, F. Cleri, and L. Colombo, J. Chem. Phys. 136, 154906 (2012).

${ }^{38}$ Manca, F., Giordano, S., Palla, P.L., Cleri, F., Colombo, L., J. Chem. Phys. 137, 244907 (2012).

${ }^{39}$ A. Rosa, T. X. Hoang, D. Marenduzzo, and A. Maritan, Biophys. Chem. 115, 251 (2005).

${ }^{40}$ T. Su and P. K. Purohit, J. Mech. Phys. Solids 58, 164 (2010).

${ }^{41}$ J. Kierfeld, O. Niamploy, V. Sa-Yakanit, and R. Lipowsky, Eur. Phys. J. E 14, 17 (2004).

${ }^{42}$ M. Rief, J. M. Fernandez, H. E. Gaub, Phys. Rev. Lett. 81, 4764 (1998).

${ }^{43}$ H. J. Kreuzer, S. H. Payne, Phys. Rev. E 63, 021906 (2001).

${ }^{44}$ Manca, F., Giordano, S., Palla, P.L., Cleri, F., Colombo, L., Phys. Rev. E 87, 032705 (2013).

${ }^{45}$ S. Giordano, Continuum Mech. Thermodyn. 30, 459 (2017).

${ }^{46}$ M. S. Kellermayer, S. B. Smith, H. L. Granzier, and C. Bustamante, Science 276, 1112 (1997).

${ }^{47}$ N. Bosaeus, A. H. El-Sagheer, T. Brown, S. B. Smith, B. Akerman, C. Bustamante, and B. Nordén, Proc. Natl. Acad. Sci. USA 109, 15179 (2012).

${ }^{48}$ J. Wang, T. B. Kouznetsova, R. Boulatov, and S. L. Craig, Nature Comm. 7, 13433 (2016).

${ }^{49}$ M. Rief, F. Oesterhelt, B. Heymann, and H. E. Gaub, Science 275, 28 (1997).

${ }^{50}$ M. Rief, M. Gautel, F. Oesterhelt, J. M. Fernandez, and H. E. Gaub, Science 276, 1109 (1997).

${ }^{51}$ M. Rief, J. Pascual, M. Saraste, and H. E. Gaub, J. Mol. Biol. 286, 553 (1999).

${ }^{52}$ F. Manca, S. Giordano, P. L. Palla, F. Cleri and L. Colombo, J. Phys.: Conf. Ser. 383, 012016 (2012).

${ }^{53}$ F. Manca, S. Giordano, P. L. Palla, F. Cleri, and L. Colombo, J. Chem. Phys. 138, 157102 (2013).

${ }^{54}$ Manca, F., Giordano, S., Palla, P.L., Cleri, F., Phys. A Stat. Mech. its Appl. 395, 154 (2014).

${ }^{55}$ R.G. Winkler, Soft Matter 6, 6183 (2010).

${ }^{56}$ I. Rouzina, and V. A. Bloomfield, Biophys. J. 80, 882 (2001).

${ }^{57}$ I. Rouzina, and V. A. Bloomfield, Biophys. J. 80, 894 (2001).

${ }^{58}$ A. E. B. Pupo, F. Falo, and A. Fiasconaro, J. Chem. Phys. 139, 095101 (2013).

${ }^{59}$ S. Cocco, J. Yan, J.-F. Léger, D. Chatenay, and J. F. Marko, Phys. Rev. E 70, 011910 (2004).

${ }^{60}$ S. Cocco, J.F. Marko, R. Monasson, A. Sarkar, and J. Yan, Eur. Phys. J. E 10, 249 (2003).

${ }^{61}$ F. Hanke and H. J. Kreuzer, Eur. Phys. J. E 22, 163 (2007).

${ }^{62}$ D. B. Staple, S. H. Payne, A. L. C. Reddin, and H. J. Kreuzer, Phys. Biol. 6, 025005 (2009).

${ }^{63}$ A. Prados, A. Carpio, and L. L. Bonilla, Phys. Rev. E 88, 012704 (2013).

${ }^{64}$ L. L. Bonilla, A. Carpio, and A. Prados, Phys. Rev. E 91, 052712 (2015).

${ }^{65}$ D. De Tommasi, N. Millardi, G. Puglisi, and G. Saccoamandi, J. R. Soc. Interface 10, 20130651 (2013). 
${ }^{66}$ D. E. Makarov, Biophys. J. 96, 2160 (2009).

${ }^{67}$ C. Guardiani, and F. Bagnoli, J. Chem. Phys. 125, 084908 (2006).

${ }^{68}$ G. Puglisi and L. Truskinovsky, J. Mech. Phys. Sol. 48, 1 (2000).

${ }^{69}$ M. Caruel, J.-M. Allain, and L. Truskinovsky, J. Mech. Phys. Sol. 76, 237 (2015).

${ }^{70}$ Y. R. Efendiev and L. Truskinovsky, Continuum Mech. Thermodyn. 22, 679 (2010).

${ }^{71}$ I. Benichou, S. Givli, J. Mech. Phys. Sol. 61, 94 (2013).

${ }^{72}$ A. F. Huxley and R. M. Simmons, Nature 233, 533 (1971).

${ }^{73}$ T. L. Hill, Proc. Nat. Acad. Sci. 70, 2732 (1973).

${ }^{74}$ M. Caruel, J.-M. Allain, and L. Truskinovsky, Phys. Rev. Lett. 110, 248103 (2013).

${ }^{75}$ M. Caruel, and L. Truskinovsky, Phys. Rev. E 93, 062407 (2016).

${ }^{76}$ S. Giordano, Soft Matter 13, 6877 (2017).

${ }^{77}$ M. Caruel and L. Truskinovsky, Rep. Prog. Phys. 81 036602 (2018).

${ }^{78}$ H. A. Kramers, Physica (The Hague) 7, 284 (1940).

${ }^{79}$ I. Benichou, S. Givli, Phys. Rev. Lett. 114, 095504 (2015).

${ }^{80}$ I. Benichou, Y. Zhang, O. K. Dudko, S. Givli, J. Mech. Phys. Sol. 95, 44 (2016).

${ }^{81}$ I. D. Vladescu, M. J. McCauley, I. Rouzina, and M. C. Williams, Phys. Rev. Lett. 95, 158102 (2005).

${ }^{82}$ C. A. Plata, F. Cecconi, M. Chinappi, and A. Prados, J. Stat. Mech. 2015, P08003 (2015).

${ }^{83}$ P. Malhotra, and J. B. Udgaonkar, Protein Sci. 25, 1924 (2016).

${ }^{84}$ R. Law, P. Carl, S. Harper, P. Dalhaimer, D. W. Speicher, and D. E. Discher, Biophys. J. 84, 533 (2003).

${ }^{85}$ T. Xu, H. Lannon, S. Wolf, F. Nakamura, and J. Brujic, Biophys. J. 104, 2022 (2013).

${ }^{86}$ D. Barrick, D. U. Ferreiro, and E. A. Komives, Curr. Opin. Struct. Biol. 18, 27 (2008).

${ }^{87}$ P. Bechtluft, R. G. H. van Leeuwen, M. Tyreman, D. Tomkiewicz, N. Nouwen, H. L. Tepper, A. J. M. Driessen, S. J. Tans, Science 318, 1458 (2007).

${ }^{88}$ A. Mashaghi, G. Kramer, P. Bechtluft, B. ZachmannBrand, A. J. M. Driessen, B. Bukau, and S. J. Tans,
Nature 500, 98 (2013).

${ }^{89} \mathrm{~J}$. W. Gibbs, Elementary principles in statistical mechanics (Charles Scribner's Sons, New York, 1902).

${ }^{90}$ A. M. Skvortsov, L. I. Klushin, F. A. M. Leermakers, J. Chem. Phys. 126, 024905 (2007).

${ }^{91}$ D.I. Dimitrov, L.I. Klushin, A.M. Skvortsov, A. Milchev, K. Binder, Eur. Phys. J. E 29, 9 (2009).

${ }^{92}$ A.M. Skvortsov, L.I. Klushin, A.A. Polotsy, K. Binder, Phys. Rev. E 85, 031803 (2012).

${ }^{93}$ I. S. Gradshteyn and I. M. Ryzhik, Table of Integrals, Series and Products (Academic Press, San Diego, 1965).

${ }^{94}$ M. Abramowitz and I. A. Stegun, Handbook of Mathematical Functions (Dover Publication Inc., New York, 1970).

${ }^{95}$ M. Radiom, P. Maroni, and M. Borkovec, ACS Macro Lett. 6, 1052 (2017).

${ }^{96}$ K. F. Wang, X. C. Pang, and S. X. Cui, Langmuir 29, 4315 (2013).

${ }^{97}$ T. Hugel, M. Grosholz, H. Clausen-Schaumann, A. Pfau, H. Gaub, and M. Seitz, Macromolecules 34, 1039 (2001).

${ }^{98}$ T. Hugel, M. Rief, M. Seitz, H. E. Gaub, and R. R. Netz, Phys. Rev. Lett. 94, 048301 (2005).

${ }^{99}$ M. Radiom and M. Borkovec, Phys. Rev. E 96, 062501 (2017).

${ }^{100}$ N. K. Balabaev and T. N. Khazanovich, Russian Journal of Physical Chemistry B 3, 242 (2009).

${ }^{101}$ G. I. Bell, Science 200, 618 (1978).

${ }^{102}$ L. Rayleigh, Phil. Mag. 37, 321 (1919).

${ }^{103}$ G. Polya, Berechnung eines bestimmten integrals, Math. Ann. 74, 204 (1913).

${ }^{104}$ L. R. G. Treloar, Trans. Faraday Soc. 42, 77 (1946).

${ }^{105}$ M. C. Wang, and E. Guth, J. Chem. Phys. 20, 1144 (1952).

${ }^{106}$ Amir A. Zadpoor, Mater. Horiz. 3, 371 (2016).

${ }^{107}$ Y. Hu, T. Lindstrøm, B. Øksendal, J. Ubøe, and T. Zhang, Inverse Powers of White Noise, Proceedings of Symposia in Pure Mathematics, Vol. 57: Stochastic Analysis (Michael C. Cranston, Mark A. Pinsky, Eds.), American Mathematical Society (1995).

${ }^{108}$ M. Schlierf and M. Rief, Biophys. J. 90, L33 (2006).

${ }^{109}$ O. K. Dudko, Quarterly Reviews of Biophysics 49, 1 (2016) 